Canad. J. Math. Vol. 73 (5), 2021 pp. 1205-1238

http://dx.doi.org/10.4153/S0008414X20000425

(c) Canadian Mathematical Society 2020. This is an Open Access article, distributed

under the terms of the Creative Commons Attribution licence (http://creativecommons.org/licenses/ by/4.0/), which permits unrestricted re-use, distribution, and reproduction in any medium, provided the original work is properly cited.

\title{
Sigma-Prikry forcing I: The Axioms
}

\author{
Alejandro Poveda, Assaf Rinot, and Dima Sinapova
}

\begin{abstract}
We introduce a class of notions of forcing which we call $\Sigma$-Prikry, and show that many of the known Prikry-type notions of forcing that centers around singular cardinals of countable cofinality are $\Sigma$-Prikry. We show that given a $\Sigma$-Prikry poset $\mathbb{P}$ and a name for a non-reflecting stationary set $T$, there exists a corresponding $\Sigma$-Prikry poset that projects to $\mathbb{P}$ and kills the stationarity of $T$. Then, in a sequel to this paper, we develop an iteration scheme for $\Sigma$-Prikry posets. Putting the two works together, we obtain a proof of the following.

Theorem. If $\kappa$ is the limit of a countable increasing sequence of supercompact cardinals, then there exists a forcing extension in which $\kappa$ remains a strong limit cardinal, every finite collection of stationary subsets of $\kappa^{+}$reflects simultaneously, and $2^{\kappa}=\kappa^{++}$.
\end{abstract}

\section{Introduction}

In $[2,3]$, Cohen invented the method of forcing as a mean to prove the independence of mathematical propositions from ZFC (the Zermelo-Fraenkel axioms for set theory). With this method, one starts with an arbitrary (transitive) model $\mathbb{M}$ of $\mathrm{ZFC}$, define there a partial order $\mathbb{P}$, and then pass to a forcing extension $\mathbb{M}[G]$ in which a new $\mathbb{P}$-generic set $G$ is adjoined. The outcome $\mathbb{M}[G]$ is the smallest model of ZFC to contain all the elements of $\mathbb{M}$, as well as the object $G$. For instance, in Cohen's celebrated work on the Continuum Hypothesis $\left(\mathrm{CH}\right.$, asserting that $\left.2^{\aleph_{0}}=\aleph_{1}\right)$, he takes $\mathbb{M}$ to be Gödel's model [15] of $\mathrm{ZFC}+\mathrm{CH}$, and defines $\mathbb{P}$ in a way that ensures that any $\mathbb{P}$-generic set $G$ will consist of $\aleph_{2}$ many distinct real numbers. Finally, to verify that " $2 \aleph^{\aleph_{0}} \geq \aleph_{2}$ " indeed holds in $\mathbb{M}[G]$, Cohen proves that $\aleph_{2}$, the second uncountable cardinal of $\mathbb{M}$, remains the second uncountable cardinal of $\mathbb{M}[G]$. In fact, Cohen proves that $\mathbb{P}$ satisfies the countable chain condition $(c c c)$ and shows that this condition ensures that the cardinals structure of $\mathbb{M}[G]$ is identical to that of $\mathbb{M}$.

Now, let us consider a proposition $\varphi$ slightly more involved than $\mathrm{CH}$, say, $\varphi$ is of the form "every uncountable group having property $p$, has property $q$, as well." Suppose that $\mathbb{M}$ is a model in which there is an uncountable group $A$ that forms a counterexample to $\varphi$. Then we could try to cook up a poset $\mathbb{P}_{A}$ such that for any

Received by the editors September 7, 2019.

Published online on Cambridge Core May 26, 2020.

Poveda was partially supported by the Spanish Government under grant MTM2017-86777-P, by Generalitat de Catalunya (Catalan Government) under grant SGR 270-2017 and by MECD Grant FPU15/00026. Rinot was partially supported by the European Research Council (grant agreement ERC-2018-StG 802756) and by the Israel Science Foundation (grant agreement 2066/18). Sinapova was partially supported by the National Science Foundation, Career-1454945.

AMS subject classification: 03E35, 03E04.

Keywords: Sigma-Prikry forcing, stationary reflection, singular cardinals hypothesis. 
$\mathbb{P}_{A}$-generic set $G$, either $G$ witness in $\mathbb{M}[G]$ that $A$ has property $q$, or $G$ witnesses in $\mathbb{M}[G]$ that $A$ ceased to have property $p$. This will solve our problem $\varphi$ for $A$, but it is very likely that in our new model $\mathbb{M}[G]$ there are other (possibly new) counterexamples to $\varphi$, meaning that we need to fix yet another counterexample $A^{\prime}$ and pass to a forcing extension $\mathbb{M}[G][H]$ solving the problem for $A^{\prime}$, and basically "keep going." But will we ever catch our tail?

It is clear that to have a chance to catch our tail, there is a need for a transfinite forcing iteration. However, unless various conditions are met, such a forcing iteration will ruin the cardinals structure, leading to a meaningless solution of the problem $\varphi$, in the sense that all uncountable groups from the intermediate models will become countable at the final model.

The first successful transfinite iteration scheme was devised by Solovay and Tennenbaum in [31], who solved a problem concerning a particular type of linear orders of size $\aleph_{1}$ known as Souslin lines. They found a natural $c c c$ poset $\mathbb{P}_{L}$ to "kill" a given Souslin line $L$, proved that a (finite-support) iteration of $c c c$ posets is again $c c c$, and proved that in an iteration of length $\aleph_{2}$, any Souslin line in the final model must show up in one of the intermediate models, meaning that they can ensure that, in their final model, there are no Souslin lines.

The Solovay-Tennenbaum technique is very useful (see [10]), but it admits no generalizations that allow to tackle problems concerning objects of size $>\aleph_{1}$. One crucial reason for the lack of generalizations has to do with the poor behavior of the higher analogues of $c c c$ at the level of cardinals $>\aleph_{1}$ (see $[17,19,20]$ for a discussion and counterexamples).

Still, various iteration schemes for posets having strong forms of the $\kappa^{+}$-chaincondition for $\kappa$ regular were devised in [9, 21-24, 26, 29]. In contrast, there is a dearth of works involving iterations at the level of the successor of singular cardinals.

A few ad-hoc treatments of iterations that are centered around a singular cardinal may be found in [27, Section 2], [6, Section 10] and [13, Section 1], and a more general framework is offered by [30, Section 3]. In [8], the authors took another approach in which they first pursue a forcing iteration along a successor of a regular cardinal $\kappa$, and at the very end they singularize $\kappa$ by appealing to Prikry forcing. This was then generalized to Radin forcing in [5].

In this project, we propose yet another approach, allowing to put the Prikry-type forcing at $\kappa$ as our very first step of the iteration, and then continue up to length $\kappa^{++}$ without collapsing cardinals. We do so by identifying a class of Prikry-type posets that are iterable in a sense to be made precise. The class is called $\Sigma$-Prikry, where $\Sigma=\left\langle\kappa_{n}\right|$ $n\langle\omega\rangle$ is a non-decreasing sequence of regular uncountable cardinals, converging to our cardinal $\kappa$. A member of the $\Sigma$-Prikry class is a triple $(\mathbb{P}, \ell, c)$ satisfying, among other things, the following:

- $\mathbb{P}=(P, \leq)$ is a notion of forcing;

- $1_{\mathbb{P}}$ decides the value of $\kappa^{+}$to be some cardinal $\mu$;

- $\ell: P \rightarrow \omega$ is a monotone grading function;

- $c: P \rightarrow \mu$ is a function witnessing that $\mathbb{P}$ is $\mu^{+}$-2-linked;

- $(\mathbb{P}, \ell)$ has the Complete Prikry Property. 
Here, $\mu^{+}$-2-linked is a well-known strong form of the $\mu^{+}$-chain-condition; as explained earlier, the latter would be too weak for any viable iteration scheme. In contrast, the Complete Prikry Property is a new concept that we introduce here in order to simultaneously capture two characteristic features of Prikry-type forcing: the decision by pure extension property and the strong Prikry property. The exact definition of $\Sigma$-Prikry may be found in Section 2 and a list of examples is given in Section 3.

Now, let us describe the first application of our framework. In his dissertation [25], Sharon claimed that if $\kappa$ is the limit of a strictly increasing sequence $\left\langle\kappa_{n} \mid n<\omega\right\rangle$ of supercompact cardinals, then, in some cardinals-preserving forcing extension, $\kappa$ remains a strong limit, $2^{\kappa}=\kappa^{++}$, and every stationary subset of $\kappa^{+}$reflects. Sharon's model is obtained by first blowing up the power of $\kappa$ using the forcing of [12, Section 3 ], and then carrying out an iteration of length $\kappa^{++}$to kill all non-reflecting stationary subsets of $\kappa^{+}$. However, a close inspection of Sharon's proof reveals a gap in the verification of the $\kappa^{++}$-chain-condition of the defined iteration, and, of course, such a chain condition is crucial for the existence of a bookkeeping function that would ensure the killing of each and every non-reflecting stationary subset of $\kappa^{+}$. In a very recent preprint [1], Ben-Neria, Hayut and Unger give an alternative proof of Sharon's result; their proof does not involve iterated forcing to kill the non-reflecting stationary sets and instead uses iterated ultrapowers to avoid the generation of non-reflecting stationary sets.

In this work, we show that Sharon's original approach is repairable and, in fact, falls into our framework. As a first step, we show that his notion of forcing for killing a single non-reflecting stationary set fits into the $\Sigma$-Prikry class:

Theorem 1.1 Suppose $\left(\mathbb{P}_{1}, \ell_{1}, c_{1}\right)$ is $\Sigma$-Prikry and $\dot{T}$ is a $\mathbb{P}_{1}$-name for a non-reflecting stationary subset of $E_{\omega}^{\mu}$. Then there exists a corresponding triple $\left(\mathbb{P}_{2}, \ell_{2}, c_{2}\right)$ such that:

- $\mathbb{P}_{2}$ is a notion of forcing that projects to $\mathbb{P}_{1}$; furthermore:

- $\left(\mathbb{P}_{2}, \ell_{2}, c_{2}\right)$ is $\sum$-Prikry admitting a forking projection to $\left(\mathbb{P}_{1}, \ell_{1}, c_{1}\right)$;

- $\mathbb{1}_{\mathbb{P}_{2}}$ forces that $\dot{T}$ is nonstationary.

The exact definition of forking projection may be found in Section 4, but, roughly speaking, this is a kind of projection that ensures a much better correspondence between the two $\Sigma$-Prikry triples, which later allows to iterate this procedure. In a sequel to this paper [18], we present our iteration scheme for $\Sigma$-Prikry notions of forcing, from which we obtain a correct proof of (a strong form of) Sharon's result:

Theorem 1.2 Suppose that $\left\langle\kappa_{n} \mid n<\omega\right\rangle$ is a strictly increasing sequence of Laverindestructible supercompact cardinals. Denote $\kappa:=\sup _{n<\omega} \kappa_{n}$. Then there exists a cofinality-preserving forcing extension in which $\kappa$ remains a strong limit, $2^{\kappa}=\kappa^{++}$, and every finite collection of stationary subsets of $\kappa^{+}$reflects simultaneously.

Remark 1.3 The preceding is optimal as, by Corollary 5.4 below, if $\kappa$ is an uncountable strong limit cardinal of countable cofinality, admitting a stationary set $S \subseteq \kappa^{+}$ with the property that every countable collection of stationary subsets of $S$ reflects simultaneously, then $2^{\kappa}=\kappa^{+}$. 


\subsection{Organization of this paper}

In Section 2, we define the class of $\Sigma$-Prikry forcing, define the $p$-tree and uncover some of its features. The proof that the Complete Prikry Property implies the Prikry Property and the Strong Prikry Property may be found there.

In Section 3, we present a few examples of notions of forcing that fit into the $\Sigma$ Prikry class. We also prove that this class is closed under lottery sum, but is not closed under products.

In Section 4, we define the notion of forking projection and establish that a graded poset admitting a forking projection to a $\Sigma$-Prikry poset is not far from being $\Sigma$-Prikry on its own.

In Section 5, we analyze the validity of simultaneous stationary reflection in generic extensions by $\Sigma$-Prikry forcing. As a corollary, the problem of getting a model with simultaneous stationary reflection reduces to forcing to get reflection of stationary sets concentrating on points of countable cofinality.

In Section 6, we prove Theorem 1.1. That is, we present a functor $\mathbb{A}(\cdot, \cdot)$ that, for any $\Sigma$-Prikry poset $\mathbb{P}$ and any $\mathbb{P}$-name for a nonreflecting stationary set $\dot{T}$, produces a $\Sigma$-Prikry poset $\mathbb{A}(\mathbb{P}, \dot{T})$ that admits a forking projection to $\mathbb{P}$ and kills the stationarity of $\dot{T}$.

\subsection{Notation and conventions}

Our forcing convention is that $p \leq q$ means that $p$ extends $q$. We write $\mathbb{P} \downarrow q$ for $\{p \in \mathbb{P} \mid p \leq q\}$. Denote $E_{\theta}^{\mu}:=\{\alpha<\mu \mid \operatorname{cf}(\alpha)=\theta\}$. The sets $E_{<\theta}^{\mu}$ and $E_{>\theta}^{\mu}$ are defined in a similar fashion. For a stationary subset $S$ of a regular uncountable cardinal $\mu$, we write $\operatorname{Tr}(S):=\left\{\delta \in E_{>\omega}^{\mu} \mid S \cap \delta\right.$ is stationary in $\left.\delta\right\} . H_{v}$ denotes the collection of all sets of hereditary cardinality less than $v$. For every set of ordinals $x$, we denote $\operatorname{cl}(x):=$ $\{\sup (x \cap \gamma) \mid \gamma \in$ Ord, $x \cap \gamma \neq \varnothing\}$ and $\operatorname{acc}^{+}(x):=\{\alpha<\sup (x) \mid \sup (x \cap \alpha)=\alpha>0\}$. For two sets of ordinals $x, y$, we write $x \subseteq y$ iff there exists an ordinal $\alpha$ such that $x=y \cap \alpha$.

\section{An abstract approach to Prikry-type forcing}

Definition 2.1 We say that $(\mathbb{P}, \ell)$ is a graded poset iff $\mathbb{P}=(P, \leq)$ is a poset, $\ell: P \rightarrow \omega$ is a surjection, and, for all $p \in P$ :

- For every $q \leq p, \ell(q) \geq \ell(p)$;

- There exists $q \leq p$ with $\ell(q)=\ell(p)+1$.

Convention 2.2 For a graded poset as above, we denote $P_{n}:=\{p \in P \mid \ell(p)=n\}$, $P_{n}^{p}:=\{q \in P \mid q \leq p, \ell(q)=\ell(p)+n\}$, and sometime write $q \leq^{n} p$ (and say the $q$ is an $n$-step extension of $p$ ) rather than writing $q \in P_{n}^{p}$.

Definition 2.3 Suppose that $\mathbb{P}=(P, \leq)$ is a notion of forcing with a greatest element 1 , and that $\Sigma=\left\langle\kappa_{n} \mid n<\omega\right\rangle$ is a non-decreasing sequence of regular uncountable cardinals, converging to some cardinal $\kappa$. Suppose that $\mu$ is a cardinal such that $1 \Vdash \mathbb{P} \check{\mu}=$ 
$\check{\kappa}^{+}{ }^{1}$ For functions $\ell: P \rightarrow \omega$ and $c: P \rightarrow \mu$, we say that $(\mathbb{P}, \ell, c)$ is $\Sigma$-Prikry iff all of the following hold:

(1) $(\mathbb{P}, \ell)$ is a graded poset;

(2) For all $n<\omega, \mathbb{P}_{n}:=\left(P_{n} \cup\{1\}\right.$, $\left.\leq\right)$ is $\kappa_{n}$-directed-closed; ${ }^{2}$

(3) For all $p, q \in P$, if $c(p)=c(q)$, then $P_{0}^{p} \cap P_{0}^{q}$ is non-empty;

(4) For all $p \in P, n, m<\omega$ and $q \leq^{n+m} p$, the set $\left\{r \leq^{n} p \mid q \leq^{m} r\right\}$ contains a greatest element which we denote by $m(p, q){ }^{3}$ In the special case $m=0$, we shall write $w(p, q)$ rather than $0(p, q) ;^{4}$

(5) For all $p \in P$, the set $W(p):=\{w(p, q) \mid q \leq p\}$ has size $<\mu$;

(6) For all $p^{\prime} \leq p$ in $P, q \mapsto w(p, q)$ forms an order-preserving map from $W\left(p^{\prime}\right)$ to $W(p)$

(7) Suppose that $U \subseteq P$ is a 0 -open set, i.e., $r \in U$ iff $P_{0}^{r} \subseteq U$. Then, for all $p \in P$ and $n<\omega$, there is $q \leq^{0} p$, such that, either $P_{n}^{q} \cap U=\varnothing$ or $P_{n}^{q} \subseteq U$.

Let us elaborate on the above definition.

- Here, $q$ is a "direct extension" of $p$ in the usual Prikry sense iff $q \leq 0$. Note that $q \leq^{0} w(p, q) \leq p$. Also, it is clear that if $p \leq^{n} q$ and $q \leq^{m} r$, then $p \leq^{n+m} r$.

- The sets $P_{n}^{p}$ consist of exactly the $n$-step extensions of $p$, and $P_{n}$ is the set of all conditions of "length" $n$, i.e., the $n$-step extensions of 1 . Note that, typically, $\mathbb{P}_{n}$ is not a complete suborder of $\mathbb{P}$, and that, for all $p, q \in P_{n}, p \leq q$ iff $p \leq^{0} q$. Thereby, $\mathbb{P}_{n}$ is not necessarily separative.

Convention. Whenever we talk about forcing with one of the $\mathbb{P}_{n}$ 's, we actually mean that we force with its separative quotient.

- Clause (3) is a very strong form of a chain condition, stronger than that of being $\mu^{+}$Knaster, and even stronger than the notion of being $\mu^{+}-2$-linked. Indeed, a poset $(P, \leq)$ is $\mu^{+}$-2-linked iff there exists a function $c: P \rightarrow \mu$ with the property that $c(p)=c(q)$ entails that $p$ and $q$ are compatible, whereas, here, we moreover require that such a compatibility will be witnessed by a 0 -step extension of $p$ and $q$.

Convention. To avoid encodings, we shall often times define the function $c$ as a map from $P$ to some natural set $\mathfrak{M}$ of size $\leq \mu$, instead of a map to the cardinal $\mu$ itself. In the special case that $\mu^{<\mu}=\mu$, we may as well take $\mathfrak{M}$ to be $H_{\mu}$.

- For every $p \in P$, the set $W(p)$ is called the $p$-tree. For every $n<\omega$, write $W_{n}(p):=$ $\left\{w(p, q) \mid q \in P_{n}^{p}\right\}$, and $W_{\geq n}(p):=\bigcup_{m=n}^{\infty} W_{m}(p)$. By Lemma 2.8 below, $(W(p), \geq)$ is a tree of height $\omega$ whose $n^{\text {th }}$ level is a maximal antichain in $\mathbb{P} \downarrow p$ for every $n<\omega$.

- Clause (7) is what we call the Complete Prikry Property (CPP), an analogue of the notion of a completely Ramsey subset of $[\omega]^{\omega}$. We shall soon show (Corollary 2.7 below) that it is a simultaneous generalization of the usual Prikry Property (PP) and the Strong Prikry Property (SPP).

\footnotetext{
${ }^{1}$ More explicitly, $\mathbf{l} \Vdash \mathbb{P} \check{\mu}=(\check{\kappa})^{+}$.

${ }^{2}$ That is, for every $D \in\left[P_{n} \cup\{\mathbf{I}\}\right]^{<\kappa_{n}}$ with the property that for all $p, p^{\prime} \in D$, there is $q \in D$ with $q \leq p, p^{\prime}$, there exists $r \in P_{n}$ such that $r \leq p$ for all $p \in D$.

${ }^{3}$ By convention, a greatest element, if exists, is unique.

${ }^{4}$ Note that $w(p, q)$ is the weakest extension of $p$ above $q$.
} 
Definition 2.4 Let $d: P \rightarrow \theta$ be some coloring, with $\theta$ a nonzero cardinal.

(1) $d$ is said to be 0-open iff $d(p) \in\{0, d(q)\}$ for every pair $q \leq 0$ of elements of $P$;

(2) We say that $H \subseteq P$ is a set of indiscernibles for $d$ iff, for all $p, q \in H,(\ell(p)=$ $\ell(q)) \Longrightarrow(d(p)=d(q))$.

Remark 2.5 The characteristic function $d: P \rightarrow 2$ of a subset $D \subseteq P$ is 0 -open iff $D$ is a 0 -open.

Lemma 2.6 For every $p \in P$, every cardinal $\theta$ with $\log (\theta)<\kappa_{\ell(p)}$ and every 0 -open coloring $d: P \rightarrow \theta,{ }^{5}$ there exists $q \leq{ }^{0} p$ such that $\mathbb{P} \downarrow q$ is a set of indiscernibles for $d$.

Proof Let $p \in P$ and $d: P \rightarrow \theta$ as above. Fix an infinite cardinal $\chi<\kappa_{\ell(p)}$ such that $2^{\chi} \geq \theta$. Fix an injective sequence $\vec{f}=\left\langle f_{\alpha} \mid \alpha<\theta\right\rangle$ consisting of functions from $\chi$ to 2 such that, in addition, $f_{0}$ is the constant function from $\chi$ to $\{0\}$.

Claim 2.6.1 Let $i<\chi$. The set $U_{i}:=\left\{r \in P \mid f_{d(r)}(i) \neq 0\right\}$ is 0 -open.

Proof Let $r \in U_{i}$ and $r^{\prime} \leq^{0} r$. As $r \in U_{i}, f_{d(r)}$ is not the constant function from $\chi$ to $\{0\}$, so that $d(r) \neq 0$. Since $d$ is a 0 -open coloring, it follows that $d\left(r^{\prime}\right)=d(r)$. Consequently, $r^{\prime} \in U_{i}$, as well.

Fix a bijection $e: \chi \leftrightarrow \chi \times \omega$. We construct $\mathrm{a} \leq^{0}$-decreasing sequence of conditions $\left\langle p_{\beta} \mid \beta \leq \chi\right\rangle$ by recursion, as follows.

- Let $p_{0}:=p$.

- Suppose that $\beta<\chi$ and that $\left\langle p_{\gamma} \mid \gamma \leq \beta\right\rangle$ has already been defined. Denote $(i, n):=e(\beta)$. Now, appeal to Definition 2.3(7) with $U_{i}, p_{\beta}$ and $n$ to obtain $p_{\beta+1} \leq{ }^{0} p_{\beta}$ such that, either $P_{n}^{p_{\beta+1}} \cap U_{i}=\varnothing$ or $P_{n}^{p_{\beta+1}} \subseteq U_{i}$.

- For every limit nonzero $\beta \leq \chi$ such that $\left\langle p_{\gamma} \mid \gamma<\beta\right\rangle$ has already been defined, appeal to Definition 2.3(2) to find a lower bound $p_{\beta}$ for the sequence.

At the end of the above recursion, let us put $q:=p_{\chi}$, so that $q \leq^{0} p$. We claim that $\mathbb{P} \downarrow q$ is a set of indiscernibles for $d$.

Suppose not, and pick two extensions $r, r^{\prime}$ of $q$ such that $\ell(r)=\ell\left(r^{\prime}\right)$ but $d(r) \neq$ $d\left(r^{\prime}\right)$. As $d(r) \neq d\left(r^{\prime}\right)$ and $\vec{f}$ is injective, let us fix $i<\chi$ such that $f_{d(r)}(i) \neq f_{d\left(r^{\prime}\right)}(i)$. Consequently, $\left|\left\{r, r^{\prime}\right\} \cap U_{i}\right|=1$. Now, put $n:=\ell(r)-\ell(p)$, so that $r, r^{\prime} \in P_{n}^{q}$. Set $\beta:=$ $e^{-1}(i, n)$. By the choice of $p_{\beta+1}$, then, either $P_{n}^{p_{\beta+1}} \cap U_{i}=\varnothing$ or $P_{n}^{p_{\beta+1}} \subseteq U_{i}$. As $q \leq^{0}$ $p_{\beta+1}$, we have $\left\{r, r^{\prime}\right\} \subseteq P_{n}^{p_{\beta+1}}$, contradicting the fact that $\left|\left\{r, r^{\prime}\right\} \cap U_{i}\right|=1$.

It follows that the Complete Prikry Property (CPP) implies the Prikry property (PP) as well as the Strong Prikry property (SPP).

Corollary 2.7 Let $p \in P$.

(1) Suppose $\varphi$ is a sentence in the forcing language. Then there is $q \leq^{0} p$ that decides $\varphi$;

(2) Suppose $D \subseteq P$ is a 0 -open set which is dense below $p$. Then there are $q \leq{ }^{0} p$ and $n<\omega$ such that $P_{n}^{q} \subseteq D .^{6}$

\footnotetext{
${ }^{5}$ Here, $\log (\theta)$ stands for the least cardinal $v$ to satisfy $2^{v} \geq \theta$.

${ }^{6}$ Note that if $D$ is open, then, moreover, $P_{m}^{q} \subseteq D$ for all $m \geq n$.
} 
Proof (1) Define a 0-open coloring $d: P \rightarrow 3$, by letting, for all $r \in P$,

$$
d(r):= \begin{cases}2, & \text { if } r \Vdash \neg \varphi ; \\ 1, & \text { if } r \Vdash \varphi ; \\ 0, & \text { otherwise. }\end{cases}
$$

Appeal to Lemma 2.6 with $d$ to get a corresponding $q \leq 0 p$. Towards a contradiction, suppose that $q$ does not decide $\varphi$. In other words, there exist $q_{1} \leq q$ and $q_{2} \leq q$ such that $d\left(q_{1}\right)=1$ and $d\left(q_{2}\right)=2$. By possibly iterating Clause (1) of Definition 2.3 finitely many times, we may find $r_{1} \leq q_{1}$ and $r_{2} \leq q_{2}$ such that $\ell\left(r_{1}\right)=\ell\left(r_{2}\right)$. By definition of $d$, we have $d\left(r_{1}\right)=1$ and $d\left(r_{2}\right)=2$. Finally, as $r_{1}$ and $r_{2}$ are two extensions $q$ of the same "length," $1=d\left(r_{1}\right)=d\left(r_{2}\right)=2$. This is a contradiction.

(2) Define a coloring $d: P \rightarrow 2$ via $d(r):=1$ iff $r \in D$. By Remark 2.5, we may appeal to Lemma 2.6 with $d$ to get a corresponding $q \leq 0$. As $D$ is dense, let us fix $r \in D$ extending $q$. Let $n:=\ell(r)-\ell(p)$, so that $d \uparrow P_{n}^{q}$ is constant with value $d(r)$. Recalling that $r \in D$ and the definition of $d$, we infer that $P_{n}^{q} \subseteq D$.

Lemma 2.8 (The $p$-tree) Let $p \in P$.

(1) For every $n<\omega, W_{n}(p)$ is a maximal antichain in $\mathbb{P} \downarrow p$;

(2) Every two compatible elements of $W(p)$ are comparable;

(3) For any pair $q^{\prime} \leq q$ in $W(p), q^{\prime} \in W(q)$;

(4) $c \uparrow W(p)$ is injective.

Proof (1) Clearly, $W_{0}(p)=\{p\}$ is a maximal antichain below $p$. Thus, hereafter, assume that $n>0$.

- To see that $W_{n}(p)=\left\{w(p, q) \mid q \in P_{n}^{p}\right\}$ is an antichain, suppose that $q_{1}, q_{2} \in P_{n}^{p}$ are such that $w\left(p, q_{1}\right)$ and $w\left(p, q_{2}\right)$ are compatible, as witnessed by some $q$. By Definition 2.3(1), $q \in P_{n+m}^{p}$ for some $m<\omega$. By Definition 2.3(4), then, $\left\{r \in P_{n}^{p} \mid q \leq r\right\}$ contains a greatest element, say, $r^{*}$. Let $i<2$ be arbitrary. As $q \leq w\left(p, q_{i}\right)$, it is not hard to see that $w\left(p, q_{i}\right)$ is the greatest element in $\left\{r \in P_{n}^{p} \mid q \leq r\right\}$, so that $w\left(p, q_{i}\right)=r^{*}$. Altogether, $w\left(p, q_{1}\right)=r^{*}=w\left(p, q_{2}\right)$.

- To verify maximality of the antichain $W_{n}(p)$, let $p^{\prime} \leq p$ be arbitrary. By Definition 2.3(1), let us pick some $q \in P_{n}^{p^{\prime}}$, so that $q \in P_{n+m}^{p}$ for some $m<\omega$. Then, by Definition 2.3(4), $\left\{r \in P_{n}^{p} \mid q \leq r\right\}$ contains a greatest element, say, $r^{*}$. As $w\left(p, r^{*}\right)=$ $r^{*}$, we have $r^{*} \in W_{n}(p)$. In addition, $r^{*}$ and $p^{\prime}$ are compatible, as witnessed by $q$.

(2) Suppose that $q_{0}, q_{1} \in W(p)$ are two compatible elements. Fix integers $n_{0}, n_{1}$ such that $q_{0} \in W_{n_{0}}(p)$ and $q_{1} \in W_{n_{1}}(p)$.

If $n_{0}=n_{1}$, then by Clause (1), $q_{0}=q_{1}$. Thus, without loss of generality, assume that $n_{0}<n_{1}$. Let $r^{*}$ be the greatest element of $\left\{r \in P_{n_{0}}^{p} \mid q_{1} \leq r\right\}$. Then $r^{*}=w\left(p, r^{*}\right) \in$ $W_{n_{0}}(p)$ and $q_{1}$ witnesses that $r^{*}$ is compatible with $q_{0}$. So $r^{*}$ and $q_{0}$ are compatible elements of $W_{n_{0}}(p)$, and hence $q_{1} \leq r^{*}=q_{0}$.

(3) Given $q^{\prime} \leq q$ as above, let $r^{\prime} \in P^{p}$ be such that $q^{\prime}=w\left(p, r^{\prime}\right)$. Now, to prove that $w\left(p, r^{\prime}\right) \in W(q)$, it suffices to show that $w\left(p, r^{\prime}\right)=w\left(q, r^{\prime}\right)$. Here goes:

- As $r^{\prime} \leq w\left(q, r^{\prime}\right) \leq q \leq p$, we infer that $w\left(q, r^{\prime}\right) \in\left\{s \mid r^{\prime} \leq s \leq p\right\}$, so that $w\left(q, r^{\prime}\right) \leq w\left(p, r^{\prime}\right)$.

- As $r^{\prime} \leq w\left(p, r^{\prime}\right)=q^{\prime} \leq q$, we infer that $w\left(p, r^{\prime}\right) \in\left\{s \mid r^{\prime} \leq s \leq q\right\}$, so that $w\left(p, r^{\prime}\right) \leq w\left(q, r^{\prime}\right)$. 
(4) By Definition 2.3(3), for all $q, q^{\prime} \in W(p)$, if $c(q)=c\left(q^{\prime}\right)$, then $q$ and $q^{\prime}$ are compatible, and they have the same $\ell$-value. It now follows from Clause (1) that $c \uparrow W(p)$ is injective.

Lemma 2.9 Suppose that $\bar{p} \leq p^{\prime} \leq p$ and $q \in W(\bar{p})$. Then $w(p, q)=w\left(p, w\left(p^{\prime}, q\right)\right){ }^{7}$

Proof As $\ell(w(p, q))=\ell(q)=\ell\left(w\left(p^{\prime}, q\right)\right)=\ell\left(w\left(p, w\left(p^{\prime}, q\right)\right)\right.$, we infer the existence of some $n<\omega$ such that both $w(p, q)$ and $w\left(p, w\left(p^{\prime}, q\right)\right)$ belong to $W_{n}(p)$. By Lemma 2.8(1), then, it suffices to verify that the two are compatible. And indeed, we have $q \leq w(p, q)$ and $q \leq w\left(p^{\prime}, q\right) \leq w\left(p, w\left(p^{\prime}, q\right)\right)$.

Lemma 2.10 (1) $\mathbb{P}$ does not add bounded subsets of $\kappa$;

(2) For every regular cardinal $v \geq \kappa$, if there exists $p \in P$ for which $p \Vdash_{\mathbb{P}} \mathrm{cf}(\check{v})<\check{\kappa}$, then there exists $p^{\prime} \leq p$ with $\left|W\left(p^{\prime}\right)\right| \geq v ;^{8}$

(3) Suppose $1 \Vdash \mathbb{P}$ “ $\kappa$ is singular." Then $\mu=\kappa^{+}$iff, for all $p \in P,|W(p)| \leq \kappa$.

Proof (1) Suppose that $p$ forces that $\sigma$ is a name for a subset of some $\theta<\kappa$. By possibly iterating Clause (1) of Definition 2.3 finitely many times, we may find $p^{\prime} \leq p$ with $\kappa_{\ell\left(p^{\prime}\right)}>\theta$. Denote $n:=\ell\left(p^{\prime}\right)$. Then by Corollary 2.7(1) and Definition 2.3(2), we may find a $\leq_{0}$-decreasing sequence of conditions, $\left\langle p_{\alpha} \mid \alpha \leq \theta\right\rangle$, with $p_{0} \leq{ }^{0} p^{\prime}$, such that, for each $\alpha<\theta, p_{\alpha} \mathbb{P}$-decides whether $\alpha$ belongs to $\sigma$. Then $p_{\theta}$ forces that $\sigma$ is a ground model set.

(2) Suppose $\theta, v$ are regular cardinals with $\theta<\kappa \leq v, \dot{f}$ is a $\mathbb{P}$-name for a function from $\theta$ to $v$, and $p \in P$ is a condition forcing that the image of $f$ is cofinal in $v$. Denote $n:=\ell(p)$. By Definition 2.3(1), we may assume that $\kappa_{n}>\theta$. For all $\alpha<\theta$, let $D_{\alpha}$ denote the open set of conditions below $p$ that $\mathbb{P}$-decides a value for $f(\alpha)$. As $D_{\alpha}$ is dense below $p$, by Corollary 2.7(2) and Definition 2.3(2), we may find a $\leq_{0}$ decreasing sequence of conditions $\left\langle p_{\alpha} \mid \alpha<\theta\right\rangle$, with $p_{0} \leq^{0} p$, and a sequence $\left\langle n_{\alpha}\right|$ $\alpha<\theta\rangle$ of elements of $\omega$, such that, for all $\alpha<\theta, P_{n_{\alpha}}^{p_{\alpha}} \subseteq D_{\alpha}$.

By Definition 2.3(2), let $p^{\prime}$ be a lower bound for $\left\{p_{\alpha} \mid \alpha<\theta\right\}$. Evidently, $P_{n_{\alpha}}^{p^{\prime}} \subseteq D_{\alpha}$ for every $\alpha<\theta$. Now, let

$$
A_{\alpha}:=\left\{\beta<v \mid \exists p \in P_{n_{\alpha}}^{p^{\prime}}[p \Vdash \mathbb{P} \dot{f}(\check{\alpha})=\check{\beta}]\right\} .
$$

By Lemma 2.8(1), we have $A_{\alpha}=\left\{\beta<v \mid \exists p \in W_{n_{\alpha}}\left(p^{\prime}\right)[p \Vdash \mathbb{P} \dot{f}(\check{\alpha})=\check{\beta}]\right\}$. Let $A:=$ $\bigcup_{\alpha<\theta} A_{\alpha}$. As $|A| \leq \sum_{\alpha<\theta}\left|W_{n_{\alpha}}\left(p^{\prime}\right)\right| \leq \theta \cdot\left|W\left(p^{\prime}\right)\right|$, it follows that if $\left|W\left(p^{\prime}\right)\right|<v$, then $\sup (A)<v$, and $p^{\prime}$ forces that the range of $f$ is bounded below $v$, which would form a contradiction. So $\left|W\left(p^{\prime}\right)\right| \geq v$.

(3) The forward implication follows from Definition 2.3(5).

Next, suppose that, for all $p \in P,|W(p)| \leq \kappa$. Towards a contradiction, suppose that there exist $p \in P$ forcing that $\kappa^{+}$is collapsed. Denote $v:=\kappa^{+}$. As $1 \Vdash \mathbb{P}$ " $\check{\kappa}$ is singular," this means that $p \Vdash \mathbb{P} \operatorname{cf}(\check{v})<\check{\kappa}$, contradicting Clause (2).

\footnotetext{
${ }^{7}$ For future reference, we point out that this fact relies only on clauses (1) and (4) of Definition 2.3.

${ }^{8}$ For future reference, we point out that this fact relies only on clauses (1),(2),(4) and (7) of Definition 2.3. Furthermore, we do not need to know that $\mathbf{l}$ decides a value for $\kappa^{+}$.
} 


\section{Examples}

\subsection{Vanilla Prikry}

Throughout this subsection assume that $\kappa$ is a measurable cardinal and that $\mathcal{U}$ is a normal measure over it. We shall show that the classical Prikry forcing $\mathbb{P}$ to singularize $\kappa$ to cofinality $\omega$ fits into the $\Sigma$-Prikry framework. Recall that $\mathbb{P}:=(P, \leq)$, where conditions in $P$ are pairs of the form $p=(s, A)$, with $s$ being a finite increasing sequence in $\kappa$ and $A \in \mathcal{U}$ with $\sup (s)<\min (A)$. The ordering $\leq$ is defined by $(s, A) \leq$ $(t, B)$ iff $t \subseteq s, A \subseteq B$ and $s \backslash t \subseteq B$.

Let $X \in\left[{ }^{<\omega} \kappa\right]^{\kappa}$. The diagonal intersection of a family $\left\{A_{s} \mid s \in X\right\} \subseteq \mathcal{U}$ is given by

$$
\triangle\left\{A_{s} \mid s \in X\right\}:=\left\{\alpha<\kappa \mid \forall s \in X\left(\max (s)<\alpha \rightarrow \alpha \in A_{s}\right)\right\}
$$

Since $\mathcal{U}$ is normal, $\triangle\left\{A_{s} \mid s \in X\right\} \in \mathcal{U}$.

Let $\Sigma$ be the $\omega$-sequence with constant value $\kappa$ and $\mu:=\kappa^{+}$. The notion of length associated to $\mathbb{P}, \ell: P \rightarrow \omega$, is given by $\ell(s, A):=|s|$. Finally, define $c: P \rightarrow^{<\omega} \kappa$ via $c(s, A):=s$. In the next proposition we verify that $(\mathbb{P}, \ell, c)$ is $\Sigma$-Prikry.

Proposition $3.1(\mathbb{P}, \ell, c)$ is $\Sigma$-Prikry.

Proof We go over the clauses of Definition 2.3.

(1) For $p=(s, A) \in P,\left(s^{\wedge}\langle v\rangle, A \backslash v+1\right) \in P_{1}^{p}$, for all $v \in A$. Moreover, by definition of $\leq$, if $q \leq p$ then $\ell(q) \geq \ell(p)$.

(2) Follows from the $\kappa$-completeness of $\mathcal{U}$.

(3) Let $p, q \in P$ and assume that $c(p)=c(q)=s$. Set $p:=(s, A)$ and $q:=(s, B)$. Clearly $(s, A \cap B)$ is in $P_{0}^{p} \cap P_{0}^{q}$.

(4) Let $p:=(s, A) \in P, n, m<\omega$ and $q:=(t, B) \in P_{n+m}^{p}$. Set $u:=t \uparrow(|s|+n)$. Then $r^{*}:=(u, A \backslash \max (u)+1)$ is the greatest element in $\left\{r \in P_{n}^{p} \mid q \leq r\right\}$.

(5) Let $p \in P$ and $n<\omega$. Denoting $p:=(s, A)$, we have that $W_{n}(p)=$ $\left\{\left(s^{\wedge} t, A \backslash \max (t)+1\right) \mid t \in[A]^{n}, t\right.$ is increasing $\}$. Clearly, $\left|W_{n}(p)\right|=\kappa<\mu$.

(6) Let $p^{\prime} \leq p$ and $q, q^{\prime} \in W\left(p^{\prime}\right)$ and assume $q^{\prime} \leq q$. Set $p:=(s, A), q:=(t, B)$ and $q^{\prime}:=(u, C)$. By the previous items, $w(p, q)=(t, A \backslash \max (t)+1)$ and $w\left(p, q^{\prime}\right)=$ $(u, A \backslash \max (u)+1)$ and, since $q^{\prime} \leq q$, is clear that $w\left(p, q^{\prime}\right) \leq w(p, q)$, as desired.

(7) This follows in a similar fashion to the classical proof of the SPP in [11, Lemma $1.13]$.

As a corollary, we infer that the $\Sigma$-Prikry class is not closed under products. Indeed, let $\mathcal{U}$ and $\mathcal{V}$ be normal measures over the same measurable cardinal $\kappa$ and let $\mathbb{P}$ and $\mathbb{Q}$ be the corresponding Vanilla Prikry notions of forcing. We claim that $\mathbb{P} \times \mathbb{Q}$ adds a bounded subset of $\kappa$, so that, by Lemma 2.10(1), it does not fit into the $\Sigma$-Prikry class.

Let $\vec{s}=\left\langle s_{n} \mid n<\omega\right\rangle$ and $\vec{t}=\left\langle t_{n} \mid n<\omega\right\rangle$ be pairwise generic Prikry-sequences with respect to $\mathbb{P}$ and $\mathbb{Q}$, i.e., $\vec{s}$ (resp. $\vec{t}$ ) generates a generic filter for $\mathbb{P}$ (resp. $\mathbb{Q}$ ) and furthermore $\vec{s} \notin V[\vec{t}]$ and $\vec{t} \notin V[\vec{s}]$. By mutual genericity, $X:=\left\{n \in \omega \mid s_{n}<t_{n}\right\}$ is infinite and it is also not hard to check that $X \notin V$. In particular, $\mathbb{P} \times \mathbb{Q}$ adds a real. 


\subsection{Supercompact Prikry forcing}

Let $\kappa<\lambda$ be two cardinals and assume that is $\mathcal{U}$ a $\lambda$-supercompact measure on $\mathcal{P}_{\kappa}(\lambda)$, namely, $\mathcal{U}$ is a $\kappa$-complete, normal and fine ultrafilter over $\mathcal{P}_{\kappa}(\lambda)$ (cf. [16, p. 301]). In this section we prove that $\mathbb{P}$, the Supercompact Prikry forcing with respect to $\mathcal{U}$ for singularizing $\kappa$ to cofinality $\omega$ and collapse the interval $\left[\kappa, \lambda^{<\kappa}\right]$, falls also into the $\Sigma$-Prikry framework. Recall that for $x, y \in \mathcal{P}_{\kappa}(\lambda), x<y$ iff $x \subseteq y$ and otp $(x)<$ $\operatorname{otp}(y \cap \kappa)$.

Recall that conditions are of the form $(\vec{x}, A)$, where $\vec{x}$ is a finite <-increasing sequence in $\mathcal{P}_{\kappa}(\lambda)$, called the stem of the condition, and $A \in \mathcal{U} .(\vec{x}, A) \leq(\vec{y}, B)$ iff $\vec{y} \subseteq \vec{x}, \vec{x} \backslash \vec{y} \subseteq B$ and $A \subseteq B$.

Given a set of stems $X$ the diagonal intersection of a family $\left\{A_{s} \mid s \in X\right\} \subseteq \mathcal{U}$ is given by

$$
\triangle\left\{A_{s} \mid s \in X\right\}:=\left\{y \in \mathcal{P}_{\kappa}(\lambda) \mid \forall s \in X\left(s<y \rightarrow y \in A_{s}\right)\right\} .
$$

Again, normality of $\mathcal{U}$ implies that $\triangle\left\{A_{s} \mid s \in X\right\} \in \mathcal{U}$. Also, one can prove a version of the classical Röwbottom Lemma for $\lambda$-supercompact measures.

Let $\Sigma$ be the $\omega$-sequence with constant value $\kappa$ and $\mu:=\left(\lambda^{<\kappa}\right)^{+}$. The notion of length associated to $\mathbb{P}, \ell: P \rightarrow \omega$, is given by $\ell(\vec{x}, A):=|\vec{x}|$.

Finally, define $c: P \rightarrow^{<\omega}\left(\lambda^{<\kappa}\right)$ via $c(\vec{x}, A):=\vec{x}$. Mimicking the proof of Proposition 3.1 one can prove the next proposition:

Proposition $3.2(\mathbb{P}, \ell, c)$ is $\Sigma$-Prikry.

\subsection{Diagonal Supercompact Prikry Forcing}

Here we show that the Diagonal Supercompact Prikry Forcing, due to Gitik and Sharon [14], can be regarded as a $\Sigma$-Prikry forcing. For economy of the discourse henceforth we shall refer to the Diagonal Supercompact Prikry Forcing simply as GS forcing, where the abbreviation GS stands for Gitik-Sharon.

Let $\left\langle\kappa_{n}\right| n\langle\omega\rangle$ be an increasing sequence of regular uncountable cardinals, and denote $\kappa:=\kappa_{0}$. Let $\Sigma$ be the $\omega$-sequence with constant value $\kappa$ and $\mu:=\left(\sup _{n<\omega} \kappa_{n}\right)^{+}$. Suppose that $\mathcal{U}$ is a supercompact measure on $\mathcal{P}_{\kappa}\left(\mu^{+}\right)$, and let $U_{n}$ be its projection projection onto $\mathcal{P}_{\kappa}\left(\kappa_{n}\right) .{ }^{9}$ It is routine to check that, for each $n<\omega, U_{n}$ is a $\kappa_{n}{ }^{-}$ supercompact measure over $\mathcal{P}_{\kappa}\left(\kappa_{n}\right)$.

We begin defining the universe $P$ of the GS poset $\mathbb{P}$ :

Definition 3.3 Define $P$ as the set of sequences $p=\left\langle x_{0}^{p}, \ldots, x_{n-1}^{p}, A_{n}^{p}, A_{n+1}^{p}, \ldots\right\rangle$ such that each $x_{i} \in \mathcal{P}_{\kappa}\left(\kappa_{i}\right), x_{i}<x_{i+1}$, and $A_{k} \in U_{k}$. Denote $\ell(p):=n$ and call the sequence $\left\langle x_{0}, \ldots, x_{n-1}\right\rangle$ the stem of $p$. Typically we will denote this sequence by $\operatorname{stem}(p)$. The order is the usual: we extend the stems by picking elements from the measure one sets, and then shrink the measure one sets.

\footnotetext{
${ }^{9}$ Namely, for each $X \subseteq \mathcal{P}_{\kappa}\left(\kappa_{n}\right), X \in U_{n}$ iff $\pi_{n}^{-1}[X] \in \mathcal{U}$, where $\pi_{n}$ is the standard projection between $\mathcal{P}_{\kappa}\left(\mu^{+}\right)$and $\mathcal{P}_{\kappa}\left(\kappa_{n}\right)$.
} 
Definition 3.4 Let $p=\left\langle x_{0}^{p}, \ldots, x_{n-1}^{p}, A_{n}^{p}, A_{n+1}^{p}, \ldots,\right\rangle$ in $P$. For $x \in A_{\ell(p)}^{p}, p^{\curvearrowright}\langle x\rangle$ stands for the unique condition

$$
q:=\left\langle x_{0}^{p}, \ldots, x_{\ell(p)-1}^{p}, x, B_{\ell(p)+1}^{p}, B_{\ell(p)+2}^{p}, \ldots\right\rangle,
$$

where, for each $i \geq \ell(p), B_{i}^{p}:=\left\{y \in A_{i}^{p} \mid x<y\right\}$. Similarly, for all $n \geq \ell(p)$, and any $<$-increasing $\vec{x}:=\left\langle x_{\ell(p)}, \ldots, x_{n+1}\right\rangle \in \prod_{i=\ell(p)}^{n+1} A_{i}^{p}$, we define $p^{\curvearrowright} \vec{x}$ to be the weakest extension of $p$ with stem equal to $\operatorname{stem}(p)^{-} \vec{x}$.

Note that whenever $q \leq p$, for some $\vec{x}$, we have that $q \leq^{0} p^{\wedge} \vec{x} \leq p$. I.e. this is exactly the needed notion to verify clauses (4), (5), (6) of Definition 2.3. In particular, for $q, p$ as above, $w(p, q)=p^{\curvearrowright \vec{x}}$.

Finally, define $c: P \rightarrow^{<\omega}\left(P_{\kappa}\left(\kappa^{+\omega}\right)\right)$ via

$$
c\left(\left\langle x_{0}^{p}, \ldots, x_{\ell(p)-1}^{p}, A_{\ell(p)}^{p}, A_{\ell(p)+1}^{p}, \ldots\right\rangle\right):=\left\langle x_{0}^{p}, \ldots, x_{\ell(p)-1}^{p}\right\rangle .
$$

Proposition $3.5(\mathbb{P}, \ell, c)$ is $\Sigma$-Prikry.

Proof We go over the clauses of Definition 2.3.

Clause (2) follows from the completeness of the normal measures. Clauses (1) and (3) are clear. Clauses (4), (5) follow from the above discussion. In particular for any $p, W_{n}(p)=\left\{p^{\curvearrowright} \vec{x} \mid \vec{x} \in \prod_{i=\ell(p)}^{n-1} A_{i}^{p},<\right.$-increasing $\}$, which has cardinality $\kappa_{n}$. Clause (6) follows from the definition of the ordering. And Clause (7) follows in a similar fashion to the proof of the SPP for the GS poset.

\subsection{AIM forcing}

We now consider the notion of forcing from [7]. Suppose $\mu$ is a strongly inaccessible cardinal, and $\Sigma=\left\langle\kappa_{n} \mid n<\omega\right\rangle$ is a strictly increasing sequence of $\mu$-supercompact cardinals. Denote $\kappa:=\sup _{n<\omega} \kappa_{n}$. For each $n<\omega$, let $U_{n}$ be some $\kappa_{n}$-complete fine normal ultrafilter on $P_{\kappa_{n}}(\mu)$, and for $\kappa \leq \alpha<\mu$ let $U_{n, \alpha}$ be the projection of $U_{n}$ to $P_{\kappa_{n}}(\alpha)$ via the map $x \mapsto x \cap \alpha$.

Definition 3.6 We define $(\mathbb{P}, \ell, c)$ with $\mathbb{P}=(P, \leq)$, as follows. $P$ consists of all sequences $p=\left\langle p_{n} \mid n<\omega\right\rangle$ such that for some $\ell(p)<\omega$, we have:

(1) For each $n<\ell(p), p_{n}$ is a function $f_{n}^{p}$ with $\operatorname{dom}\left(f_{n}^{p}\right) \subseteq[\kappa, \mu),\left|\operatorname{dom}\left(f_{n}^{p}\right)\right|<\mu$, and for all $\eta \in \operatorname{dom}\left(f_{n}^{p}\right), f_{n}^{p}(\eta) \in P_{\kappa_{n}}(\eta)$;

(2) For each $n \geq \ell(p), p_{n}$ is a triple $\left(a_{n}^{p}, A_{n}^{p}, f_{n}^{p}\right)$, where:

a) $a_{n}^{p}$ is a subset of $[\kappa, \mu)$ with $\left|a_{n}^{p}\right|<\mu$ that moreover admits a maximal element $\alpha_{n}^{p}$

b) $A_{n}^{p} \in U_{n, \alpha_{n}^{p}}$

c) $f_{n}^{p}$ is a function with $\operatorname{dom}\left(f_{n}^{p}\right) \subseteq[\kappa, \mu) \backslash a_{n}^{p},\left|\operatorname{dom}\left(f_{n}^{p}\right)\right|<\mu$ such that, for all $\eta \in \operatorname{dom}\left(f_{n}^{p}\right), f_{n}^{p}(\eta) \in P_{\kappa_{n}}(\eta)$.

(3) $\left\langle a_{n}^{p} \mid \ell(p) \leq n<\omega\right\rangle$ is $\subseteq$-increasing.

We let $p \leq q$ if and only if:

(1) $\ell(p) \geq \ell(q)$.

(2) For all $n, f_{n}^{p} \supseteq f_{n}^{q}$; 
(3) For $n$ with $\ell(q) \leq n<\ell(p), a_{n}^{q} \subseteq \operatorname{dom}\left(f_{n}^{p}\right), f_{n}^{p}\left(\alpha_{n}^{q}\right) \in A_{n}^{q}$, and $f_{n}^{p}(\eta)=f_{n}^{p}\left(\alpha_{n}^{q}\right) \cap$ $\eta$ for all $\eta \in a_{n}^{q} .^{10}$

(4) $\left(f_{n}^{p}\left(\alpha_{n}^{q}\right)\right)_{\ell(q) \leq n<\ell(p)}$ is $\subseteq$-increasing.

(5) For $n \geq \ell(p)$, we have $a_{n}^{q} \subseteq a_{n}^{p}$, and $x \cap \alpha_{n}^{q} \in A_{n}^{q}$ for all $x \in A_{n}^{p}$.

(6) For $n \geq \ell(p)$, if $\ell(q)<\ell(p)$, then $f_{\ell(p)-1}^{p}\left(\alpha_{\ell(p)-1}^{q}\right) \subseteq x$ for all $x \in A_{n}^{p}$.

Finally, by cardinality considerations, we find $c: P \rightarrow \mu$ which is an injection.

By virtue of Lemma 4 and Corollary 1 of [7], $\mathbb{P}$ collapses all cardinals $\theta$ with $\kappa<\theta<$ $\mu$ and makes $\mu$ the successor of $\kappa$. Next, we briefly go over the clauses of Definition 2.3 to explain why $(\mathbb{P}, \ell, c)$ is $\Sigma$-Prikry.

By the completeness of the measures, we get that for each $n, \mathbb{P}_{n}$ is $\kappa_{n}$-directedclosed giving Clause (2). Clauses (1) and (3) are clear. For Clauses (4), (5), (6) we need to recall some definitions and facts from [7].

Definition 3.7 For conditions $r \leq q$, we let $\operatorname{stem}(r, q)$ denote the finite sequence $\left(f_{i}^{r}\left(\alpha_{i}^{q}\right)\right)_{\ell(q) \leq i<\ell(r)}$.

Definition 3.8 Let $q$ be a condition. Let $l \in(\ell(q), \omega)$ and $s \in \prod_{\ell(q) \leq i<l} A_{i}^{q}$ be a $\subseteq$ increasing sequence. Define $q+s$ as the $\omega$-sequence $\left(r_{k}\right)_{k<\omega}$ such that:

- For $k<\ell(q), r_{k}=f_{k}^{q}$.

- For $\ell(q) \leq k<l, r_{k}$ is the function with domain $\operatorname{dom}\left(f_{k}^{q}\right) \cup a_{k}^{q}$ such that $r_{k}(\eta)=$ $f_{k}^{q}(\eta)$ for $\eta \in \operatorname{dom}\left(f_{k}^{q}\right)$ and $r_{k}(\eta)=s_{k} \cap \eta$ for $\eta \in a_{k}^{q}$.

- For $k \geq l, r_{k}=\left(f_{k}^{q}, a_{k}^{q}, B_{k}\right)$ where $B_{k}=\left\{x \in A_{k}^{q}: s_{l-1} \subseteq x\right\}$. ${ }^{11}$

By convention we also define $q+\langle\rangle=q$.

In [7, Lemma 8], it is shown that for $q$ and $s$ as in Definition 3.8, $q+s$ is a condition in $P$ extending $q$. Moreover, for each $r \leq q, r \leq^{0} q+\operatorname{stem}(r, q)$ and also is not hard to check that $q+\operatorname{stem}(r, q)$ is the weakest extension of $q$ above $r$; i.e., in our notation, $q+\operatorname{stem}(r, q)=w(q, r)$. Thereby, for each $n, W_{n}(q)$ is the set of all conditions of the form $q+s$, where $s \in \prod_{\ell(q) \leq i<n} A_{i}^{q}$. It thus follows that $W_{n}(q)$ has cardinality less than $\mu$, hence yielding clauses (4) and (5).

For Clause (6), let $q^{\prime} \leq q$ and $r_{0}, r_{1} \in W\left(q^{\prime}\right)$ with $r_{0} \leq r_{1}$. By the previous discussion, for each $i \in 2$, there is $s_{i}$ such that $r_{i}=q+s_{i}$ and $w\left(q, q+s_{i}\right)=q+s_{i}$. Altogether, we have shown that $w\left(q, q+s_{0}\right) \leq w\left(q, q+s_{1}\right)$, hence yielding Clause (6).

Finally, Clause (7) of Definition 2.3 follows in a similar fashion to the Prikry property arguments in [7, Lemma 10 and 11]. The main point is that given a 0 -open set $U$ and a condition $p$, for every possible $s$ as in the above definitions, we check if there is $q \leq p+s$ in $U$. If there is, call it $p_{s}$; otherwise, let $p_{s}:=p+s$. Doing this via a careful induction one constructs $q \leq^{0} p$, such that, for all $s, q+s \leq{ }^{0} p_{s}$. Then we shrink the measure one sets to ensure that either each $q+s$ is in $U$ or none is.

To sum up, we have the following:

Proposition $3.9(\mathbb{P}, \ell, c)$ is $\Sigma$-Prikry.

\footnotetext{
${ }^{10}$ This is the corresponding analogous of condition (2d) in [11, Definition 2.10] for the Extenderbased Prikry forcing. See also Subsection 3.5 below.

${ }^{11}$ Notice that $f_{l-1}^{q}\left(\alpha_{l-1}^{q}\right)=s_{l-1}$, as $s_{l-1} \subseteq \alpha_{l-1}^{q}$.
} 


\subsection{Extender-based Prikry Forcing}

Suppose that $\left\langle\kappa_{n} \mid n<\omega\right\rangle$ is an increasing sequence of regular cardinals, let $\kappa:=$ $\sup _{n<\omega} \kappa_{n}, \mu:=\kappa^{+}$and let $\lambda>\mu$ be such that $\lambda^{<\lambda}=\lambda$. Suppose further that each $\kappa_{n}$ carries a $\left(\kappa_{n}, \lambda+1\right)$-extender $E_{n}:=\left\langle E_{n, \alpha} \mid \alpha<\lambda\right\rangle$. Then extender-based Prikry forcing with respect to these extenders, denoted by $\mathbb{P}_{\text {ebpf }}$, adds sequences $\left\langle f_{n} \mid n<\omega\right\rangle$, where each $f_{n}: \lambda \rightarrow \kappa_{n}$ is generic for the Cohen forcing $\operatorname{Add}(\mu, \lambda)$, and an unbounded set $F \subset \lambda$ with the following properties:

- setting $t_{\alpha} \in \prod_{n<\omega} \kappa_{n}$ by $t_{\alpha}(n):=f_{n}(\alpha)$, we have that $t_{\alpha} \notin V$ iff $\alpha \in F$;

- for all $\alpha<\beta$ both in $F$, for all large $n, t_{\alpha}(n)<t_{\beta}(n)$;

- for all $\alpha \in F, t_{\alpha}$ is a Prikry generic sequence with respect to the measures $\left\langle E_{n, \alpha}\right|$ $n<\omega\rangle$ (i.e. for all measure one sets from these ultrafilters, the sequence meets them on a tail end)

In particular, forcing with $\mathbb{P}_{\text {ebpf }}$ makes $2^{\kappa}=\lambda$. This forcing plays an important role in the proof of Theorem 1.2. In a sequel to this paper [18], we will describe this forcing in detail and prove that it is $\Sigma$-Prikry, where $\Sigma:=\left\langle\kappa_{n} \mid n<\omega\right\rangle$.

\subsection{Lottery sum}

Suppose that $\Sigma=\left\langle\kappa_{n} \mid n<\omega\right\rangle$ is non-decreasing sequence of regular uncountable cardinals, converging to some cardinal $\kappa, \mu$ is a cardinal, and $\left\langle\left(\mathbb{Q}_{i}, \ell_{i}, c_{i}\right) \mid i<v\right\rangle$ is a sequence of $\Sigma$-Prikry notions of forcing such that $v<\mu$ and, for all $i<v, 1_{\mathbb{Q}_{i}} \Vdash_{\mathbb{Q}_{i}}$ $\check{\mu}=\check{\kappa}^{+}$.

Define $P:=\left\{(i, p) \mid i<v, p \in Q_{i}\right\} \cup\{\varnothing\}$ and an ordering $\leq$, letting $(i, p) \leq(j, q)$ iff $i=j$ and $p \leq \mathbb{Q}_{i} q$, as well as setting $x \leq \varnothing$ for any $x \in P$. Set $\mathbb{P}:=(P, \leq)$ and note that $1_{\mathbb{P}}=\varnothing$ and $\mathbb{1}_{\mathbb{P}} \Vdash_{\mathbb{Q}_{i}} \check{\mu}=\check{\kappa}^{+}$. Now, define $\ell: P \rightarrow \omega$ by letting $\ell(\varnothing):=0$ and $\ell(i, p):=$ $\ell_{i}(p)$. Finally, define $c: P \rightarrow \mu \times \mu$ by letting $c(\varnothing):=(0,0)$ and $c(i, p):=\left(i, c_{i}(p)\right)$.

Proposition $3.10(\mathbb{P}, \ell, c)$ is $\Sigma$-Prikry.

Proof We go over the clauses of Definition 2.3.

(1) As $(i, q) \leq(j, p)$ entails $i=j$ and $q \leq_{\mathbb{Q}_{i}} p$, we infer from the fact that $\left(\mathbb{Q}_{i}, \ell_{i}, c_{i}\right)$ is $\Sigma$-Prikry, that $\ell(i, p)=\ell(p) \leq \ell(q)=\ell(i, q)$.

(2) Let $D \in\left[P_{n} \cup\{\varnothing\}\right]^{<\kappa_{n}}$ be directed. Find $i<v$ such that $D \backslash\{\varnothing\} \subseteq\{i\} \times$ $\left(Q_{i}\right)_{n}$. Now, as $\left(\mathbb{Q}_{i}, \ell_{i}, c_{i}\right)$ is $\Sigma$-Prikry, there exists a lower bound $p$ for $\left\{q \in\left(Q_{i}\right)_{n} \mid(i, q) \in D\right\}$. Evidently, $(i, p)$ is a lower bound for $D$.

(3) Follows from the fact that, for all $i<v,\left(\mathbb{Q}_{i}, \ell_{i}, c_{i}\right)$ is $\Sigma$-Prikry.

(4)-(5) Let $x \in P$ and $(i, q) \leq x$. If $x=\varnothing$ it is not hard to check that $w(\varnothing, \varnothing)=\varnothing$ and that, more generally, $m(\varnothing,(i, q))=\left(i, m\left(1_{\mathbb{Q}_{i}}, q\right)\right)$. Hence, $W(\varnothing) \subseteq\{\varnothing\} \cup$ $\cup_{i<v} W\left(1_{\mathbb{Q}_{i}}\right)$. Analogously if $x \neq \varnothing$, say $x=(i, p)$, then $m((i, p),(i, q))=$ $(i, m(p, q))$ and thus, in particular, $W_{n}(i, p)=\{i\} \times W_{n}(p)$. Since $v<\mu$, this yields clauses (4) and (5).

(6) This is obvious.

(7) Let $U \subseteq P$ be a 0 -open set and fix $x \in P$ and $n<\omega$. If $x \neq \varnothing$, denote $(i, p):=x$. Otherwise, let $(i, p):=\left(0,1_{\mathbb{P}_{0}}\right)$. In both cases, $(i, p) \leq^{0} x$. Now, it is not hard to check that $U_{i}:=\left\{q \in Q_{i} \mid(i, q) \in U\right\}$ is also 0 -open. Since $\left(\mathbb{Q}_{i}, \ell_{i}, c_{i}\right)$ is 
$\Sigma$-Prikry we may find $q \in\left(Q_{i}\right)_{0}^{p}$ such that either $\left(Q_{i}\right)_{n}^{q} \subseteq U_{i}$ or $\left(Q_{i}\right)_{n}^{q} \cap U_{i}=$ $\varnothing$. Set $y:=(i, q)$. Clearly $y \leq^{0} x$. If $P_{n}^{q} \cap U \neq \varnothing$ then clearly $\left(Q_{i}\right)_{n}^{q} \cap U_{i} \neq \varnothing$, hence $\left(Q_{i}\right)_{n}^{q} \subseteq U_{i}$, and thus $P_{n}^{q} \subseteq U$.

\section{Forking projections}

In this section, we introduce the notion of forking projection which will play a key role in Section 6.

Definition 4.1 Suppose that $\left(\mathbb{P}, \ell_{\mathbb{P}}, c_{\mathbb{P}}\right)$ is a $\sum$-Prikry triple, $\mathbb{A}=(A, \unlhd)$ is a notion of forcing, and $\ell_{\mathbb{A}}$ and $c_{\mathbb{A}}$ are functions with $\operatorname{dom}\left(\ell_{\mathbb{A}}\right)=\operatorname{dom}\left(c_{\mathbb{A}}\right)=A$.

A pair of functions $(h, \pi)$ is said to be a forking projection from $\left(\mathbb{A}, \ell_{\mathbb{A}}\right)$ to $\left(\mathbb{P}, \ell_{\mathbb{P}}\right)$ iff all of the following hold:

(1) $\pi$ is a projection from $\mathbb{A}$ onto $\mathbb{P}$, and $\ell_{\mathbb{A}}=\ell_{\mathbb{P}} \circ \pi$;

(2) for all $a \in A, \downarrow(a)$ is an order-preserving function from $(\mathbb{P} \downarrow \pi(a), \leq)$ to $(\mathbb{A} \downarrow a, \unlhd)$;

(3) for all $p \in P,\{a \in A \mid \pi(a)=p\}$ admits a greatest element, which we denote by $\lceil p\rceil^{\mathbb{A}}$

(4) for all $n, m<\omega$ and $b \unlhd^{n+m} a, m(a, b)$ exists and satisfies:

$$
m(a, b)=\pitchfork(a)(m(\pi(a), \pi(b)))
$$

(5) for all $a \in A$ and $r \leq \pi(a), \pi(\pitchfork(a)(r))=r$;

(6) for all $a \in A$ and $r \leq \pi(a), a=\lceil\pi(a)\rceil^{\mathbb{A}}$ iff $h(a)(r)=\lceil r\rceil^{\mathbb{A}}$;

(7) for all $a \in A, a^{\prime} \unlhd^{0} a$ and $r \leq^{0} \pi\left(a^{\prime}\right)$, $\pitchfork\left(a^{\prime}\right)(r) \unlhd \pitchfork(a)(r)$.

The pair $(h, \pi)$ is said to be a forking projection from $\left(\mathbb{A}, \ell_{\mathbb{A}}, c_{\mathbb{A}}\right)$ to $\left(\mathbb{P}, \ell_{\mathbb{P}}, c_{\mathbb{P}}\right)$ iff, in addition to all of the above, the following holds:

(8) for all $a, a^{\prime} \in A$, if $c_{\mathbb{A}}(a)=c_{\mathbb{A}}\left(a^{\prime}\right)$, then $c_{\mathbb{P}}(\pi(a))=c_{\mathbb{P}}\left(\pi\left(a^{\prime}\right)\right)$ and, for all $r \in$ $P_{0}^{\pi(a)} \cap P_{0}^{\pi\left(a^{\prime}\right)}, \pitchfork(a)(r)=\pitchfork\left(a^{\prime}\right)(r)$.

Example 4.2 Suppose that $\left(\mathbb{P}, \ell_{\mathbb{P}}, c_{\mathbb{P}}\right)$ is any $\Sigma$-Prikry triple and that $\mathbb{Q}$ is any notion of forcing with a greatest element $1_{\mathbb{Q}}$. Let $\mathbb{A}=(A, \unlhd)$ be the product forcing $\mathbb{P} \times \mathbb{Q}$. Define $\pi: A \rightarrow P$ via $\pi(p, q):=p$, and, for each $a=(p, q)$ in $A$, define $\pitchfork(a):$ $\mathbb{P} \downarrow p \rightarrow \mathbb{A} \downarrow a$ via $\downarrow(a)(r):=(r, q)$. Set $\ell_{\mathbb{A}}:=\ell_{\mathbb{P}} \circ \pi$. Define $c_{\mathbb{A}}: A \rightarrow \operatorname{Im}\left(c_{\mathbb{P}}\right) \times Q$ via $c_{\mathbb{A}}(p, q):=\left(c_{\mathbb{P}}(p), q\right)$. Then $\lceil p\rceil^{\mathbb{A}}=\left(p, 1_{\mathbb{Q}}\right), w\left((p, q),\left(p^{\prime}, q^{\prime}\right)\right)=\left(w\left(p, p^{\prime}\right), q\right)$, and the pair $(h, \pi)$ is a forking projection from $\left(\mathbb{A}, \ell_{\mathbb{A}}, c_{\mathbb{A}}\right)$ to $\left(\mathbb{P}, \ell_{\mathbb{P}}, c_{\mathbb{P}}\right)$.

Lemma 4.3 Suppose that $(h, \pi)$ is a forking projection from $\left(\mathbb{A}, \ell_{\mathbb{A}}\right)$ to $\left(\mathbb{P}, \ell_{\mathbb{P}}\right)$. Let $a \in A$.

(1) $\pitchfork(a) \uparrow W(\pi(a))$ forms a bijection from $W(\pi(a))$ to $W(a)$;

(2) for all $n<\omega$ and $r \leq^{n} \pi(a)$, $\pitchfork(a)(r) \in A_{n}^{a}$.

Proof (1) By Clauses (4) and (5) of Definition 4.1.

(2) By Clauses (1), (2) and (5) of Definition 4.1.

Lemma 4.4 Suppose that $(h, \pi)$ is a forking projection from $\left(\mathbb{A}, \ell_{\mathbb{A}}\right)$ to $\left(\mathbb{P}, \ell_{\mathbb{P}}\right)$. Let $U \subseteq A$ and $a \in A$. Denote $U_{a}:=U \cap(\mathbb{A} \downarrow a)$. 
(1) If $U_{a}$ is 0-open, then so is $\pi\left[U_{a}\right]$;

(2) If $U_{a}$ is dense below $a$, then $\pi\left[U_{a}\right]$ is dense below $\pi(a)$.

Proof (1) Suppose $U_{a}$ is 0 -open. To see that $\pi\left[U_{a}\right]$ is 0 -open, let $p \in \pi\left[U_{a}\right]$ and $p^{\prime} \leq^{0} p$ be arbitrary. Find $b \in U_{a}$ such that $\pi(b)=p$ and set $b^{\prime}:=\pitchfork(b)\left(p^{\prime}\right)$. Clearly, $b^{\prime}$ is well-defined and by Definition 4.1(5), $b^{\prime} \unlhd^{0} b$, so that, by 0 -openness of $U_{a}$, $b^{\prime} \in U_{a}$. Again, Definition 4.1(5) yields $\pi\left(b^{\prime}\right)=\pi\left(\pitchfork(b)\left(p^{\prime}\right)\right)=p^{\prime}$, thus $p^{\prime} \in \pi\left[U_{a}\right]$, as desired.

(2) Suppose that $U_{a}$ is dense below $a$. To see that $\pi\left[U_{a}\right]$ is dense below $\pi(a)$, let $p \leq \pi(a)$ be arbitrary. Since, by Definition 4.1(1), $\pi$ is a projection from $\mathbb{A}$ to $\mathbb{P}$, we may find $a^{*} \unlhd a$ such that $\pi\left(a^{*}\right) \leq p$. As $U_{a}$ is dense below $a$, we may then find $a^{\star} \unlhd a^{*}$ in $U_{a}$. Clearly, $\pi\left(a^{\star}\right) \leq p$.

Throughout the rest of this section, suppose that:

- $\mathbb{P}=(P, \leq)$ is a notion of forcing with a greatest element $\mathbb{1}_{\mathbb{P}}$;

- $\mathbb{A}=(A, \unlhd)$ is a notion of forcing with a greatest element $1_{\mathbb{A}}$;

- $\Sigma=\left\langle\kappa_{n} \mid n<\omega\right\rangle$ is a non-decreasing sequence of regular uncountable cardinals, converging to some cardinal $\kappa$, and $\mu$ is a cardinal such that $\mathbb{1}_{\mathbb{P}} \Vdash \mathbb{P} \check{\mu}=\check{\kappa}^{+}$;

- $\ell_{\mathbb{P}}$ and $c_{\mathbb{P}}$ are functions witnessing that $\left(\mathbb{P}, \ell_{\mathbb{P}}, c_{\mathbb{P}}\right)$ is a $\Sigma$-Prikry;

- $\ell_{\mathbb{A}}$ and $c_{\mathbb{A}}$ are functions with $\operatorname{dom}\left(\ell_{\mathbb{A}}\right)=\operatorname{dom}\left(c_{\mathbb{A}}\right)=A$;

- $(h, \pi)$ is a forking projection from $\left(\mathbb{A}, \ell_{\mathbb{A}}, c_{\mathbb{A}}\right)$ to $\left(\mathbb{P}, \ell_{\mathbb{P}}, c_{\mathbb{P}}\right)$.

We shall now go over each of the clauses of Definition 2.3 and collect sufficient conditions for the triple $\left(\mathbb{A}, \ell_{\mathbb{A}}, c_{\mathbb{A}}\right)$ to be $\Sigma$-Prikry, as well.

Lemma $4.5\left(\mathbb{A}, \ell_{\mathbb{A}}\right)$ is a graded poset.

Proof For all $a, b \in A, \quad b \unlhd a \Longrightarrow \pi(b) \leq \pi(a) \Longrightarrow \ell_{\mathbb{A}}(b)=\ell_{\mathbb{P}}(\pi(b)) \geq \ell_{\mathbb{P}}$ $(\pi(a))=\ell_{\mathbb{A}}(a)$. In addition, as $\left(\mathbb{P}, \ell_{\mathbb{P}}\right)$ is a graded poset, for any given $a \in A$, we may pick $r \in P_{1}^{\pi(a)}$. By Lemma 4.3(2), then, $\pitchfork(a)(r)$ witnesses that $A_{1}^{a}$ is non-empty.

Lemma 4.6 Let $n<\omega$. Suppose that for every directed family $D$ of conditions in $\mathbb{A}_{n}$ with $|D|<\kappa_{n}$, if the map $d \mapsto \pi(d)$ is constant over $D$, then $D$ admits a lower bound in $\mathbb{A}_{n}$.

Then $\mathbb{A}_{n}$ is $\kappa_{n}$-directed-closed.

Proof Suppose that $E$ is a given directed family in $\mathbb{A}_{n}$ of size less than $\kappa_{n}$. In particular, $\{\pi(e) \mid e \in E\}$ is a directed family in $\mathbb{P}_{n}$ of size less than $\kappa_{n}$; hence, by Definition 2.3(2), we may find a lower bound for it (in $\left.\mathbb{P}_{n}\right)$, say, $r$. Put $D:=\{\hbar(e)(r) \mid$ $e \in E\}$. By Lemma 4.3(2), $D$ is a family of conditions in $\mathbb{A}_{n}$ with $|D|<\kappa_{n}$. By Definition 4.1(5), the map $d \mapsto \pi(d)$ is constant (indeed, with value $r$ ) over $D$.

Claim 4.6.1 $D$ is directed.

Proof Given $d_{0}, d_{1} \in D$, fix $e_{0}, e_{1} \in E$ such that $d_{i}=\pitchfork\left(e_{i}\right)(r)$ for all $i<2$. As $E$ is directed, let us pick $e^{*} \in E$ such that $e^{*} \unlhd e_{0}, e_{1}$. Put $d^{*}:=\pitchfork\left(e^{*}\right)(r)$, so that $d^{*} \in D$. Then, by Definition 4.1(7), $d^{*} \unlhd d_{0}, d_{1}$. 
Now, by the hypothesis of the lemma, we may pick a lower bound for $D$ (in $\mathbb{A}_{n}$ ), say, $b$. By Definition 4.1(2), for all $a \in E, b \unlhd \pitchfork(a)(r) \unlhd a$, and hence $b$ is a lower bound for $E$.

Lemma 4.7 For all $a, a^{\prime} \in A$, if $c_{\mathbb{A}}(a)=c_{\mathbb{A}}\left(a^{\prime}\right)$, then $A_{0}^{a} \cap A_{0}^{a^{\prime}}$ is non-empty.

In particular, if $\left|\operatorname{Im}\left(c_{\mathbb{A}}\right)\right| \leq \mu$, then $\mathbb{A}$ is $\mu^{+}$-2-linked.

Proof By Definition 4.1(8), $c(\pi(a))=c\left(\pi\left(a^{\prime}\right)\right)$. Since $\left(\mathbb{P}, \ell_{\mathbb{P}}, c_{\mathbb{P}}\right)$ is $\Sigma$-Prikry, Definition 2.3(3) guarantees the existence of some $r \in P_{0}^{\pi(a)} \cap P_{0}^{\pi\left(a^{\prime}\right)}$ and thus, again by Definition 4.1(8), $\pitchfork(a)(r)=\pitchfork\left(a^{\prime}\right)(r)$. Finally, Lemma 4.3(2) yields that this common value is in $A_{0}^{a} \cap A_{0}^{a^{\prime}}$, as desired.

Lemma 4.8 For all $a \in A, n, m<\omega$ and $b \unlhd^{n+m} a, m(a, b)$ exists.

Proof This is covered by Definition 4.1(4).

Lemma 4.9 For all $a \in A,|W(a)|<\mu$.

Proof This follows from Lemma 4.3(1) and Definition 2.3(5) for $\left(\mathbb{P}, \ell_{\mathbb{P}}, c_{\mathbb{P}}\right)$.

Lemma 4.10 For all $a^{\prime} \leq a$ in $A, b \mapsto w(a, b)$ forms an order-preserving map from $W\left(a^{\prime}\right)$ to $W(a)$.

Proof Fix an arbitrary pair $b^{\prime} \unlhd b$ in $W\left(a^{\prime}\right)$, and let us show that $w\left(a, b^{\prime}\right) \unlhd w(a, b)$. By Definition 4.1(4) with $m=0, w\left(a, b^{\prime}\right)=\pitchfork(a)\left(w\left(\pi(a), \pi\left(b^{\prime}\right)\right)\right)$ and $w(a, b)=$ $h(a)(w(\pi(a), \pi(b)))$. On the other hand, $\pi$ is a projection, in particular orderpreserving, hence $\pi\left(b^{\prime}\right) \leq \pi(b)$, and also both such conditions extend $\pi(a)$. By Definition 2.3(6) for $\left(\mathbb{P}, \ell_{\mathbb{P}}, c_{\mathbb{P}}\right), w\left(\pi(a), \pi\left(b^{\prime}\right)\right) \leq w(\pi(a), \pi(b))$, and thus, appealing to Definition 4.1(7), it follows that

$$
\pitchfork(a)\left(w\left(\pi(a), \pi\left(b^{\prime}\right)\right)\right) \unlhd \pitchfork(a)(w(\pi(a), \pi(b))),
$$

which yields the desired result.

Definition 4.11 The forking projection $(h, \pi)$ is said to have the mixing property iff for all $a \in A, n<\omega, q \leq^{0} \pi(a)$, and a function $g: W_{n}(q) \rightarrow \mathbb{A} \downarrow a$ such that $\pi \circ g$ is the identity map, ${ }^{12}$ there exists $b \unlhd^{0} a$ with $\pi(b)=q$ such that $\pitchfork(b)(r) \unlhd^{0} g(r)$ for every $r \in W_{n}(q)$.

Lemma 4.12 Suppose that $(h, \pi)$ has the mixing property. Let $U \subseteq A$ be a 0-open set. Then, for all $a \in A$ and $n<\omega$, there is $b \unlhd^{0}$ a such that, either $A_{n}^{b} \cap U=\varnothing$ or $A_{n}^{b} \subseteq U$.

Proof Let $a \in A$ and $n<\omega$. Set $U_{a}:=U \cap(\mathbb{A} \downarrow a), \bar{U}:=\pi\left[U_{a}\right]$, and $p:=\pi(a)$. By Lemma $4.4(1), \bar{U}$ is 0 -open. Since $\left(\mathbb{P}, \ell_{\mathbb{P}}, c_{\mathbb{P}}\right)$ is $\sum$-Prikry, we now appeal to Definition 2.3(7) and find $q \leq^{0} p$ such that, either $P_{n}^{q} \cap \bar{U}=\varnothing$ or $P_{n}^{q} \subseteq \bar{U}$.

Claim 4.12.1 If $P_{n}^{q} \cap \bar{U}=\varnothing$, then there exists $b \unlhd^{0} a$ with $\pi(b)=q$ such that $A_{n}^{b} \cap$ $U=\varnothing$.

\footnotetext{
${ }^{12}$ Equivalently, a function $g: W_{n}(q) \rightarrow A$ such that $g(r) \unlhd a$ and $\pi(g(r))=r$ for every $r \in W_{n}(q)$.
} 
Proof Suppose that $P_{n}^{q} \cap \bar{U}=\varnothing$. Set $b:=\pitchfork(a)(q)$, so that $b \unlhd a$ and $\pi(b)=q$. As $\ell_{\mathbb{A}}(b)=\ell_{\mathbb{P}}(q)=\ell_{\mathbb{A}}(a)$, we moreover have $b \unlhd^{0} a$. Finally, since $d \in A_{n}^{b} \cap U \Longrightarrow$ $\pi(d) \in P_{n}^{q} \cap \bar{U}$, we infer that $A_{n}^{b} \cap U=\varnothing$.

Claim 4.12.2 If $P_{n}^{q} \subseteq \bar{U}$, then there exists $b \unlhd^{0} a$ with $\pi(b)=q$ such that $A_{n}^{b} \subseteq U$.

Proof Suppose that $P_{n}^{q} \subseteq \bar{U}$. So, for every $r \in P_{n}^{q}$, we may pick $a_{r} \in U_{a}$ such that $\pi\left(a_{r}\right)=r$. Define a function $g: W_{n}(q) \rightarrow U_{a}$ via $g(r):=a_{r}$. By the mixing property, we now obtain a condition $b \unlhd^{0} a$ such that $\pitchfork(b)(r) \unlhd^{0} g(r)$ for every $r \in W_{n}(q)$. As $U$ is 0-open, it follows that $\pitchfork(b)$ “ $W_{n}(q) \subseteq U$. By Lemma 4.3(1), $W_{n}(b)=\pitchfork(b)$ “ $W_{n}(q) \subseteq$ $U$; hence, again by 0 -openess of $U, A_{n}^{b} \subseteq U$, as desired.

This completes the proof.

Corollary 4.13 Suppose that Clauses $(2)$ and $(7)$ of Definition 2.3 are valid for $\left(\mathbb{A}, \ell_{\mathbb{A}}\right)$. If $1_{\mathbb{P}} \vdash_{\mathbb{P}}$ “ $\check{\kappa}$ is singular, " then $1_{\mathbb{A}} \vdash_{\mathbb{A}} \check{\mu}=\check{\kappa}^{+}$.

Proof Suppose that $1_{\mathbb{A}} \Vdash \mathbb{A} \check{A}=\check{\kappa}^{+}$. As $1_{\mathbb{P}} \Vdash_{\mathbb{P}} \check{\mu}=\check{\kappa}^{+}$and $\mathbb{A}$ projects to $\mathbb{P}$, this means that there exists $a \in A$ such that $a \Vdash_{\mathbb{A}}|\check{\mu}| \leq|\check{\kappa}|$. Towards a contradiction, suppose that $\mathbb{1}_{\mathbb{P}} \Vdash_{\mathbb{P}}$ “ $\check{\kappa}$ is singular." As $\mathbb{A}$ projects to $\mathbb{P}$, it altogether follows that $a \Vdash_{\mathbb{A}} \operatorname{cf}(\check{\mu})<$ $\check{\kappa}$. By Lemma 2.10(2), then, there exists $a^{\prime} \unlhd a$ with $\left|W\left(a^{\prime}\right)\right| \geq \mu$, contradicting Lemma 4.8(2).

\section{Simultaneous stationary reflection}

Definition 5.1 For cardinals $\theta<\mu=\operatorname{cf}(\mu)$, and stationary subsets $S, T$ of $\mu$, the principle $\operatorname{Refl}(<\theta, S, T)$ asserts that for every collection $\mathcal{S}$ of stationary subsets of $S$, with $|\mathcal{S}|<\theta$ and $\sup (\{\operatorname{cf}(\alpha) \mid \alpha \in \bigcup \mathcal{S}\})<\sup (S)$, the set $T \cap \bigcap_{\mathcal{S} \in \mathcal{S}} \cap \operatorname{Tr}(S)$ is nonempty.

We write $\operatorname{Refl}(<\theta, S)$ for $\operatorname{Refl}(<\theta, S, \mu)$ and $\operatorname{Refl}(\theta, S)$ for $\operatorname{Refl}\left(<\theta^{+}, S\right) .{ }^{13}$

Definition 5.2 (Shelah, [28, Definition 5.1, p. 85]) For infinite cardinals $\mu \geq v \geq \theta$, define

$$
\operatorname{cov}(\mu, v, \theta, 2):=\min \left\{|\mathcal{A}| \mid \mathcal{A} \subseteq[\mu]^{<v} \forall X \in[\mu]^{<\theta} \exists A \in \mathcal{A}(X \subseteq A)\right\} .
$$

The following proposition is implicit in the work of Solovay on the Singular Cardinal Hypothesis (SCH).

Proposition 5.3 Suppose $\operatorname{Refl}\left(<\theta, S, E_{<v}^{\mu}\right)$ holds for a stationary $S \subseteq \mu$ and some cardinal $v \in \mu$. Then $\operatorname{cov}(\mu, v, \theta, 2)=\mu$.

Proof Let $\left\langle S_{i}\right| i\langle\mu\rangle$ be a partition of $S$ into mutually disjoint stationary sets. Put $T:=\{\alpha<\mu \mid \omega<\operatorname{cf}(\alpha)<v\}$. Set $\mathcal{A}:=\left\{A_{\alpha} \mid \alpha \in T\right\}$, where for each $\alpha \in T, A_{\alpha}:=\{i<$ $\mu \mid S_{i} \cap \alpha$ is stationary $\}$. Since each $\alpha \in T$ admits a club $C_{\alpha}$ of order-type $<v$, and $C_{\alpha} \cap$ $S_{i} \neq \varnothing$ for all $i \in A_{\alpha}$, while $S_{i} \cap S_{j}=\varnothing$ for all $i<j<\mu$, we get that $\mathcal{A} \subseteq[\mu]^{<v}$.

$\operatorname{By} \operatorname{Refl}\left(<\theta, S, E_{<\nu}^{\mu}\right)$, for every $X \in[\mu]^{<\theta}$, there must exist some $A \in \mathcal{A}$ such that $X \subseteq A$. Altogether, $\mathcal{A}$ witnesses that $\operatorname{cov}(\mu, v, \theta, 2)=\mu$.

\footnotetext{
${ }^{13}$ Where, for $\theta$ finite, $\theta^{+}$stands for $\theta+1$.
} 
Note that for every singular strong limit $\kappa, \operatorname{cov}\left(\kappa^{+}, \kappa,(\operatorname{cf}(\kappa))^{+}, 2\right)=2^{\kappa}$. In particular:

Corollary 5.4 If $\kappa$ is a singular strong limit cardinal admitting a stationary subset $S \subseteq$ $\kappa^{+}$for which $\operatorname{Refl}(\operatorname{cf}(\kappa), S)$ holds, then $2^{\kappa}=\kappa^{+}$.

Throughout the rest of this section, suppose that $(\mathbb{P}, \ell, c)$ is a given $\Sigma$-Prikry notion of forcing. Denote $\mathbb{P}=(P, \leq)$ and $\Sigma=\left\langle\kappa_{n} \mid n<\omega\right\rangle$. Also, define $\kappa$ and $\mu$ as in Definition 2.3. Our universe of sets is denoted by $V$, and we write $\Gamma:=\{\alpha<\mu \mid$ $\left.\omega<\operatorname{cf}^{V}(\alpha)<\kappa\right\} .14$

Lemma 5.5 Suppose that $r^{*} \in P$ and that $\tau$ is a $\mathbb{P}$-name. For all $n<\omega$, write $\dot{T}_{n}:=$ $\left\{(\check{\alpha}, p) \mid(\alpha, p) \in \mu \times P_{n} \& p \Vdash \mathbb{P} \check{\alpha} \in \tau\right\}$. Then one of the following holds:

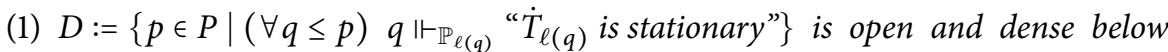
$r^{*} ; 15$

(2) There exist $r^{\star} \leq r^{\star}$ and $I \in[\omega]^{\omega}$ such that, for all $q \leq r^{\star}$ with $\ell(q) \in I$,

$$
q \Vdash_{\mathbb{P}_{\ell(q)}} \text { “ } \dot{T}_{\ell(q)} \text { is nonstationary." }
$$

Proof $D$ is clearly open. Suppose that $D$ is not dense below $r^{*}$. Then, we may pick some condition $p^{*} \leq r^{*}$ such that, for all $p \leq p^{*}$, there is $q \leq p$, such that $q \psi_{\mathbb{P}_{\ell(q)}}$ “ $\dot{T}_{\ell(q)}$ is stationary," i.e., there exists $q^{\prime} \leq q$ in $\mathbb{P}_{\ell(q)}$ such that $q^{\prime} \Vdash_{\mathbb{P}_{\ell(q)}}$ " $T_{\ell(q)}$ is nonstationary." Hence, for all $p \leq p^{*}$, there is $q^{\prime} \leq p$, such that $q^{\prime} \Vdash_{\mathbb{P}_{\ell(q)}}$ " $\dot{T}_{\ell(q)}$ is nonstationary." In other words, the 0 -open set $E:=\left\{q \in \mathbb{P} \mid q \Vdash \mathbb{P}_{\ell(q)}\right.$ " $\dot{T}_{\ell(q)}$ is nonstationary" $\}$ is dense below $p^{*}$.

Now, define a 0 -open coloring $d: P \rightarrow 2$ via $d(q):=1$ iff $q \in E$. By virtue of Lemma 2.6, find $r^{\star} \leq^{0} p^{\star}$ such that $\mathbb{P} \downarrow r^{\star}$ is a set of indiscernibles for $d$. Note that as $E$ is dense below $r^{\star}$, Clause (1) of Definition 2.3 entails that the set $I:=\left\{\ell\left(q^{\prime}\right) \mid\right.$ $\left.q^{\prime} \leq r^{\star} \& q^{\prime} \in E\right\}$ must be infinite. Finally, as $\mathbb{P} \downarrow r^{\star}$ is a set of indiscernibles for $d$, for all $q \leq r^{\star}$ with $\ell(q) \in I$, we indeed have $q \in E$.

Lemma 5.6 Suppose that $r^{\star} \in P, I \in[\omega]^{\omega}$, and $\left\langle\dot{C}_{n} \mid n \in I\right\rangle$ is a sequence such that, for all $q \leq r^{\star}$ with $\ell(q) \in I$, we have:

$$
q \Vdash_{\mathbb{P}_{\ell(q)}} \text { " } \dot{C}_{\ell(q)} \text { is a club in } \check{\mu} . "
$$

Consider the $\mathbb{P}$-name $\dot{Y}:=\{(\check{\alpha}, q) \mid(\alpha, q) \in R\}$, where

$$
R:=\left\{(\alpha, q) \in \mu \times P \mid q \leq r^{\star} \& \forall r \leq q\left[\ell(r) \in I \rightarrow r \Vdash_{\mathbb{P}_{\ell(r)}} \check{\alpha} \in \dot{C}_{\ell(r)}\right]\right\} .
$$

Suppose $G$ is $\mathbb{P}$-generic over $V$, with $r^{\star} \in G$. Let $Y$ be the interpretation of $\dot{Y}$ in $V[G]$. Then:

(1) $V[G] \vDash Y$ is unbounded in $\mu$;

(2) $V[G] \vDash \operatorname{acc}^{+}(Y) \cap \Gamma \subseteq Y$.

Proof We commence with a claim.

\footnotetext{
${ }^{14}$ All findings of the analysis in this section goes through if we replace $\mu$ by a regular cardinal $v \geq \mu$ and replace $\Gamma$ by $\left\{\alpha<v \mid \omega<\mathrm{cf}^{V}(\alpha)<\kappa\right\}$.

${ }^{15}$ Recall that we identify each of the $\mathbb{P}_{n}$ 's with its separative quotient.
} 
Claim 5.6.1 For every $p \leq r^{\star}$ and $\gamma<\mu$, there exist $\bar{p} \leq^{0} p$ and $\bar{\gamma} \in(\gamma, \mu)$ such that, for every $q \leq \bar{p}$ with $\ell(q) \in I, q \Vdash_{\mathbb{P}_{\ell(q)}}$ " $\dot{C}_{\ell(q)} \cap(\gamma, \bar{\gamma})$ is non-empty."

Proof Given $p$ and $\gamma$ as above, write:

$$
D_{p, \gamma}:=\left\{q \in \mathbb{P} \mid q \leq p \& \ell(q) \in I \& \exists \gamma^{\prime}>\gamma\left(q \Vdash_{\mathbb{P}_{\ell(q)}} \check{\gamma}^{\prime} \in \dot{C}_{\ell(q)}\right)\right\} .
$$

Note that $I_{p, \gamma}:=\left\{\ell(q) \mid q \in D_{p, \gamma}\right\}$ is equal to $I \backslash \ell(p) .{ }^{16}$ Let $d: P \rightarrow 2$ be defined via $d(r):=1$ iff $r \in D_{p, \gamma}$. As $D_{p, \gamma}$ is 0 -open we get from Lemma 2.6 a condition $\bar{p} \leq^{0} p$ such that $\mathbb{P} \downarrow \bar{p}$ is a set of indiscernibles for $d$. Thereby, for all $n<\omega$, if $P_{n}^{\bar{p}} \cap D_{p, \gamma} \neq \varnothing$, then $P_{n}^{\bar{p}} \subseteq D_{p, \gamma}$. As $\bar{p} \leq p, I_{p, \gamma}=I \backslash \ell(p)$, and $W_{n}(\bar{p}) \subseteq P_{n}^{\bar{p}}$ for all $n<\omega$, we get in particular that $A_{n}:=W_{n-\ell(\bar{p})}(\bar{p})$ is a subset of $D_{p, \gamma}$ for all $n \in I \backslash \ell(p)$.

For all $n \in I \backslash \ell(p)$ and $r \in A_{n}$, fix $\gamma_{r} \in(\gamma, \mu)$ such that

$$
r \Vdash_{\mathbb{P}_{\ell(r)}} \gamma_{r} \in \dot{C}_{\ell(r)} .
$$
is $<\mu$.

By Definition 2.3(5), $\left|\bigcup_{n \in I \backslash \ell(p)} A_{n}\right|<\mu$, so that $\bar{\gamma}:=\sup \left\{\gamma_{r} \mid r \in \bigcup_{n \in I \backslash \ell(p)} A_{n}\right\}+1$

Now, let $q \leq \bar{p}$ with length in $I$ be arbitrary. As $I_{p, \gamma}=I \backslash \ell(p)$, we have $\ell(q) \in I_{p, \gamma}$. In particular, $P_{\ell(q)-\ell(\bar{p})}^{\bar{p}} \cap D_{p, \gamma} \neq \varnothing$, and thus $A_{\ell(q)} \subseteq D_{p, \gamma}$. Pick $r \in A_{\ell(q)}$ with $q \leq r$. Then $r \Vdash_{\mathbb{P}_{\ell(r)}} \gamma_{r} \in \dot{C}_{\ell(r)}$. In particular, $q \Vdash_{\ell(q)}$ " $\dot{C}_{\ell(q)} \cap(\gamma, \bar{\gamma})$ is non-empty." is

Now, let $G$ be a $\mathbb{P}$-generic with $r^{\star} \in G$. Of course, the interpretation of $\dot{Y}$ in $V[G]$

$$
Y:=\left\{\alpha<\mu \mid(\exists q \in G)(\forall r \leq q)\left[\ell(r) \in I \rightarrow r \Vdash_{\mathbb{P}_{\ell(r)}} \check{\alpha} \in \dot{C}_{\ell(r)}\right]\right\} .
$$

Claim 5.6.2 (1) $Y$ is unbounded in $V[G]$;

(2) $\operatorname{acc}^{+}(Y) \cap \Gamma \subseteq Y$.

Proof (1) We run a density argument in $V$. Let $p \leq r^{\star}$ and $\gamma<\mu$ be arbitrary. By an iterative application of Claim 5.6.1, we find a $\leq_{0}$-decreasing sequence of conditions in $\mathbb{P},\left\langle p_{n} \mid n<\omega\right\rangle$, and an increasing sequence of ordinals below $\mu,\left\langle\gamma_{n} \mid n<\omega\right\rangle$, such that $p_{0} \leq^{0} p, \gamma_{0}=\gamma$, and such that for every $n<\omega$ and every $q \leq p_{n}$ with $\ell(q) \in I$, we have that $q \Vdash_{\mathbb{P}_{\ell(q)}}$ " $\dot{C}_{\ell(q)} \cap\left(\gamma_{n}, \gamma_{n+1}\right)$ is non-empty."

By Definition 2.3(2), $\mathbb{P}_{\ell(p)}$ is $\sigma$-closed, so let $q^{*}$ be a lower bound for $\left\langle q_{n} \mid n<\omega\right\rangle$. Put $\gamma^{*}:=\sup _{n<\omega} \gamma_{n}$. Then for every $r \leq q^{*}$ with length in $I$, we have $r \Vdash_{\mathbb{P}_{\ell(r)}} \gamma^{*} \in \dot{C}_{\ell(r)}$. That is, $q^{*}$ witnesses that $\gamma^{*} \in Y \backslash \gamma$.

(2) Suppose that $\alpha \in \operatorname{acc}^{+}(Y) \cap \Gamma$. Set $\eta:=\operatorname{cf}^{V}(\alpha)$, and pick a large enough $k<\omega$ such that $\eta<\kappa_{k}$. Fix $p \in G$ such that $p \leq r^{\star}, p \Vdash \check{\alpha} \in \operatorname{acc}^{+}(\dot{Y})$, and $\ell(p) \geq k$.

Work in $V$. Let $\left\langle\alpha_{j} \mid j<\eta\right\rangle$ be an increasing cofinal sequence in $\alpha$. For each $j<\eta$, consider the set $D_{j}:=\left\{q \in P \mid \exists \gamma \in\left(\alpha_{j}, \alpha\right) q \Vdash_{\mathbb{P}} \check{\gamma} \in \dot{Y}\right\}$. Clearly, $D_{j}$ is open and dense below $p$. We claim that the intersection $\bigcap_{j<\eta} D_{j}$ is dense below $p$, as well. To this end, let $p^{\prime} \leq p$ be arbitrary. For each $j<\eta, D_{j}$ is 0 -open and dense below $p^{\prime}$, so since $\eta<\kappa_{k} \leq \kappa_{\ell\left(p^{\prime}\right)}$, we obtain from Corollary 2.7(2) and Definition 2.3(2), a $\leq_{0}$-decreasing

\footnotetext{
${ }^{16}$ By standard facts about forcing, if $\mathbb{Q}$ is a notion of forcing, and $q$ is a condition in $\mathbb{Q}$ forcing that $\dot{C}$ is some cofinal subset of a cardinal $\mu$, then for every ordinal $\gamma<\mu$, there exists an extension $q^{\prime}$ of $q$ and some ordinal $\gamma^{\prime}$ above $\gamma$ such that $q^{\prime} \Vdash \mathbb{Q} \check{\gamma}^{\prime} \in \dot{C}$.
} 
sequence $\left\langle q_{j} \mid j \leq \eta\right\rangle$ along with a sequence of natural numbers $\left\langle n_{j} \mid j<\eta\right\rangle$ such that $q_{0} \leq{ }^{0} p^{\prime}$ and $P_{n_{j}}^{q_{j}} \subseteq D_{j}$ for all $j<\eta$. Let $p^{\prime \prime}:=q_{\eta}$. As $\eta=\operatorname{cf}^{V}(\alpha)>\omega$, we may pick a cofinal $J \subseteq \eta$ for which $\left\{n_{j} \mid j \in J\right\}$ is a singleton, say, $\{n\}$. Then $P_{n}^{p^{\prime \prime}} \subseteq \bigcap_{j \in J} P_{n_{j}}^{q_{j}} \subseteq$ $\bigcap_{j \in J} D_{j}=\bigcap_{j<\eta} D_{j}$. Thus, the latter contains an element extending $p^{\prime \prime}$, which extends $p^{\prime}$.

Fix $q \in G \cap \bigcap_{j<\eta} D_{j}$ extending $p$ and let us show that $q$ witnesses that $\alpha$ is in $Y$. That is, we shall verify that, for all $r \leq q$ with $\ell(r) \in I, r \Vdash_{\mathbb{P}_{\ell(r)}} \check{\alpha} \in \dot{C}_{\ell(r)}$. First, notice that for all $j<\eta$, there exists some $\gamma_{j} \in\left(\alpha_{j}, \alpha\right)$ such that $q \Vdash \mathbb{P} \check{\gamma}_{j} \in \dot{Y}$. Now let $r \leq q$ with $\ell(r) \in I$ be arbitrary and notice that $r \Vdash_{\mathbb{P}_{\ell(r)}} \check{\gamma}_{j} \in \dot{C}_{\ell(r)}$ for all $j<\eta$, hence $r \Vdash_{\mathbb{P}_{\ell(r)}}$ $\check{\alpha} \in \dot{C}_{\ell(r)}$.

This completes the proof of Lemma 5.6.

Lemma 5.7 Suppose that $r^{*} \in P$ forces that $\tau$ is a $\mathbb{P}$-name for a stationary subset $T$ of $\Gamma$. For all $n<\omega$, write $\dot{T}_{n}:=\left\{(\check{\alpha}, p) \mid(\alpha, p) \in \mu \times P_{n} \& p \Vdash_{\mathbb{P}} \check{\alpha} \in \tau\right\}$. Then $D:=\{p \in P \mid$ $(\forall q \leq p) q \Vdash_{\mathbb{P}_{\ell(q)}}$ “ $\dot{T}_{\ell(q)}$ is stationary" $\}$ is open and dense below $r^{*}$.

Proof Suppose not. Then, by Lemma 5.5, let us pick $r^{\star} \leq r^{*}$ and $I \in[\omega]^{\omega}$ such that, for all $q \leq r^{\star}$ with $\ell(q) \in I$,

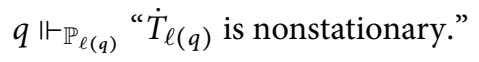

Now, for each $n \in I$, we appeal to the maximal principle (also known as the mixing lemma) to find a $\mathbb{P}_{n}$-name $\dot{C}_{n}$ for a club subset of $\mu$, such that, for all $q \leq r^{\star}$ with $\ell(q) \in I$, we have $q \mathbb{P}_{\ell(q)} \dot{C}_{\ell(q)} \cap \dot{T}_{\ell(q)}=\varnothing$. Consider the $\mathbb{P}$-name:

$$
\dot{Y}:=\left\{(\check{\alpha}, q) \in \mu \times P \mid q \leq r^{\star} \& \forall r \leq q\left[\ell(r) \in I \rightarrow r \Vdash_{\mathbb{P}_{\ell(r)}} \check{\alpha} \in \dot{C}_{\ell(r)}\right]\right\} .
$$

Let $G$ be $\mathbb{P}$-generic over $V$, with $r^{\star} \in G$, and $Y$ be the interpretation of $\dot{Y}$ in $V[G]$. By Lemma 5.6:

(1) $V[G] \vDash Y$ is unbounded in $\mu$;

(2) $V[G] \vDash \operatorname{acc}^{+}(Y) \cap \Gamma \subseteq Y$.

As $r^{\star} \leq r^{\star}$, our hypothesis entails:

(3) $V[G] \vDash T$ is a stationary subset of $\Gamma$.

So $V[G] \vDash Y \cap T \neq \varnothing$. Pick $\alpha<\mu$ and $r \in G$ such that $r \Vdash_{\mathbb{P}} \check{\alpha} \in \dot{Y} \cap \tau$. Of course, we may find such $r$ that in addition satisfies $r \leq r^{\star}$ and $\ell(r) \in I$. By definition of $\dot{T}_{\ell(r)}$, the ordered-pair $(\check{\alpha}, r)$ is an element of the name $\dot{T}_{\ell(r)}$. In particular, $r \Vdash_{\mathbb{P}_{\ell(r)}} \check{\alpha} \in \dot{T}_{\ell(r)}$.

From $r \leq r^{\star}, \ell(r) \in I$, and $r \Vdash_{\mathbb{P}} \check{\alpha} \in \dot{Y}$, we have $r \Vdash_{\mathbb{P}_{\ell(r)}} \check{\alpha} \in \dot{C}_{\ell(r)}$.

Altogether $r \Vdash_{\mathbb{P}_{\ell(r)}} \dot{C}_{\ell(r)} \cap \dot{T}_{\ell(r)} \neq \varnothing$, contradicting the choice of $\dot{C}_{\ell(r)}$.

Recall that a supercompact cardinal $\chi$ is said to be Laver-indestructible iff for every $\chi$-directed-closed notion of forcing $\mathbb{Q}, 1_{\mathbb{Q}} \Vdash \mathbb{Q}$ “ $\chi$ is supercompact." Also recall that for every supercompact cardinal $\chi$ and every regular cardinal $v \geq \chi, \operatorname{Refl}\left(<\chi, E_{<\chi}^{v}, E_{<\chi}^{v}\right)$ holds. We refer the reader to [4] for further details. For our purpose, we would just need the following: 
Lemma 5.8 For all $n<\omega$, if $\kappa_{n}$ is a Laver-indestructible supercompact cardinal, then $V^{\mathbb{P}_{n}} \vDash \operatorname{Refl}\left(<\omega, E_{<\kappa_{n}}^{\mu}, E_{<\kappa_{n}}^{\mu}\right){ }^{17}$

Proof By Definition 2.3(2), $\mathbb{P}_{n}$ is $\kappa_{n}$-directed-closed, and hence $V^{\mathbb{P}_{n}} \vDash$ “ $\kappa_{n}$ is supercompact." In particular, $V^{\mathbb{P}_{n}} \vDash \operatorname{Refl}\left(<\omega, E_{<\kappa_{n}}^{\mu}, E_{<\kappa_{n}}^{\mu}\right)$.

\section{Lemma 5.9 Suppose:}

- For all $n<\omega, V^{\mathbb{P}_{n}} \vDash \operatorname{Refl}\left(<\omega, E_{<\kappa_{n}}^{\mu}, E_{<\kappa_{n}}^{\mu}\right)$;

- $r^{*} \in P$ forces that $\left\langle\tau^{i} \mid i<k\right\rangle$ is a finite sequence of $\mathbb{P}$-names for stationary subsets of $\left(E_{<\kappa}^{\mu}\right)^{V}$;

Write $\dot{T}_{n}^{i}:=\left\{(\check{\alpha}, p) \mid(\alpha, p) \in \mu \times P_{n} \& p \Vdash \mathbb{P} \check{\alpha} \in \tau^{i}\right\}$ for all $i<k$ and $n<\omega$.

Suppose $D^{i}:=\left\{p \in P \mid(\forall q \leq p) q \Vdash_{\mathbb{P}_{\ell(q)}}\right.$ “䄪 $\ell_{\ell(q)}^{i}$ is stationary" $\}$ is open and dense below $r^{*}$ for each $i<k$. Then for every $\mathbb{P}$-generic $G$ over $V$ with $r^{*} \in G,\left\langle T^{i} \mid i<k\right\rangle$ reflects simultaneously in $V[G] .^{18}$

Proof We run a density argument below the condition $r^{*}$. Given an arbitrary $p_{0} \leq r^{*}$, pick $p \in \bigcap_{i<k} D^{i}$ below $p_{0}$ and a large enough $m<\omega$ such that $p \Vdash \mathbb{P}$ " $\forall i<$ $k\left(\tau^{i} \cap E_{<\kappa_{m}}^{\mu}\right)$ is stationary." By possibly extending $p$ using Definition 2.3(1), we may assume that $n:=\ell(p)$ is $\geq m$. Let $G_{n}$ be $\mathbb{P}_{n}$-generic with $p \in G_{n}$. As $V\left[G_{n}\right] \vDash$ $\operatorname{Refl}\left(<\omega, E_{<\kappa_{n}}^{\mu}, E_{<\kappa_{n}}^{\mu}\right)$, let us fix some $q \leq^{0} p$ in $G_{n}$, and some $\delta \in E_{<\kappa_{n}}^{\mu}$ such that $q \Vdash \mathbb{P}_{n}$ “ $\forall i<k\left(\dot{T}_{n}^{i} \cap \delta\right.$ is stationary $) . "$

In $V$, pick a club $C \subseteq \delta$ of order type $\operatorname{cf}(\delta)$. Note that $|C|<\kappa_{n}$. Then for each $i<k$, $q \Vdash \mathbb{P}_{n}$ “ $\dot{T}_{n}^{i} \cap C$ is stationary in $\delta$." Working for a moment in $V\left[G_{n}\right]$, write $A^{i}:=C \cap$ $\left(\dot{T}_{n}^{i}\right)_{G_{n}}$. Since $\mathbb{P}_{n}$ is $\kappa_{n}$-closed, we may find $r \in P_{n}$ extending $q$ that, for all $i<k$, decides $A^{i}$ to be some ground model stationary subset $B^{i}$ of $\delta$. Then, for every $i<k$,

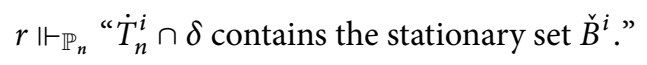

By definition of the name $\dot{T}_{n}^{i}$, we have that $r \Vdash_{\mathbb{P}} \check{B}^{i} \subseteq \tau^{i} \cap \delta$. Finally, $\operatorname{since} \operatorname{otp}\left(B^{i}\right) \leq$ $\delta<\kappa$, Lemma 2.10(1), $B^{i}$ remains stationary in $V^{\mathbb{P}}$ for each $i$. So, $r \leq p_{0}$, and $r \Vdash \mathbb{P}$ " $\tau^{i} \cap \delta$ is stationary for each $i<k$."

Corollary 5.10 Suppose $V^{\mathbb{P}_{n}} \vDash \operatorname{Refl}\left(<\omega, E_{<\kappa_{n}}^{\mu}, E_{<\kappa_{n}}^{\mu}\right)$ for all $n<\omega$. Then $V^{\mathbb{P}} \vDash$ $\operatorname{Refl}(<\omega, \Gamma)$.

Proof Let $r^{*}$ be a condition in $G$ forcing that $\left\langle\tau^{i} \mid i<k\right\rangle$ is a finite sequence of $\mathbb{P}$ names for stationary subsets $\left\langle T^{i} \mid i<k\right\rangle$ of $\Gamma$. For each $i<k$ and each $n<\omega$, write $\dot{T}_{n}^{i}:=\left\{(\check{\alpha}, p) \mid(\alpha, p) \in\left(\mu \times P_{n}\right) \& p \Vdash \mathbb{P} \check{\alpha} \in \tau^{i}\right\}$. By Lemma 5.7, for each $i<k, D^{i}:=$ $\left\{p \in P \mid(\forall q \leq p) q \Vdash_{\mathbb{P}_{\ell(q)}}\right.$ " $\dot{T}_{\ell(q)}^{i}$ is stationary" $\}$ is open and dense below $r^{*}$. Finally, by virtue of Lemma 5.9, $\left\langle T^{i} \mid i<k\right\rangle$ reflects simultaneously in $V[G]$. sion.

Putting Lemma 5.8 together with Corollary 5.10, we arrive at the following conclu-

Corollary 5.11 Suppose that each cardinal in $\Sigma$ is a Laver-indestructible supercompact cardinal. Then $1 \Vdash \mathbb{P} \operatorname{Refl}(<\omega, \Gamma)$.

\footnotetext{
${ }^{17}$ Note that, as $\mathbb{P}_{n}$ is $\kappa_{n}$-closed, $\left(E_{<\kappa_{n}}^{\mu}\right)^{V^{\mathbb{P}} n}=\left(E_{<\kappa_{n}}^{\mu}\right)^{V}$.

${ }^{18}\left\langle T^{i} \mid i<k\right\rangle$ stands for the $G$-interpretation of the sequence of $\mathbb{P}$-names $\left\langle\tau^{i} \mid i<k\right\rangle$.
} 
Towards a model $V[G]$ satisfying $\operatorname{Refl}\left(<\omega, \kappa^{+}\right)$, we would need to address the reflection of stationary subsets of $\mu \backslash \Gamma$. In the special case that $\kappa$ is singular and $\mu=\kappa^{+}$, the set $\mu \backslash \Gamma$ will be nothing but $\left(E_{\omega}^{\mu}\right)^{V}$. It is not hard to verify that in this scenario, $V[G]$ will satisfy $\operatorname{Refl}\left(<\omega, \kappa^{+}\right)$iff it will satisfy $\operatorname{Refl}(<\omega, \Gamma)+\operatorname{Refl}\left(1,\left(E_{\omega}^{\mu}\right)^{V}, \Gamma\right) \cdot{ }^{19}$ For this, in the next section we shall devise a notion of forcing for killing a given single counterexample to $\operatorname{Refl}\left(1, E_{\omega}^{\mu}, \Gamma\right)$. Then, in [18], we find a mean to iterate it.

\section{Killing one non-reflecting stationary set}

Throughout this section, suppose that $(\mathbb{P}, \ell, c)$ is a given $\Sigma$-Prikry notion of forcing. Denote $\mathbb{P}=(P, \leq)$ and $\Sigma=\left\langle\kappa_{n} \mid n<\omega\right\rangle$. Also, define $\kappa$ and $\mu$ as in Definition 2.3, and assume that $1_{\mathbb{P}} \Vdash_{\mathbb{P}}$ “ $\check{\kappa}$ is singular" and that $\mu^{<\mu}=\mu$. Our universe of sets is denoted by $V$, and we assume that, for all $n<\omega, V^{\mathbb{P}_{n}} \vDash \operatorname{Refl}\left(1, E_{\omega}^{\mu}, E_{<\kappa_{n}}^{\mu}\right) .{ }^{20}$ Write $\Gamma:=\{\alpha<\mu \mid$ $\left.\omega<\mathrm{cf}^{V}(\alpha)<\kappa\right\}$.

Lemma 6.1 Suppose $r^{\star} \in P$ forces that $\dot{T}$ is a $\mathbb{P}$-name for a stationary subset $T$ of $\left(E_{\omega}^{\mu}\right)^{V}$ that does not reflect in $\Gamma$. For each $n<\omega$, write $\dot{T}_{n}:=\left\{(\check{\alpha}, p) \mid(\alpha, p) \in E_{\omega}^{\mu} \times P_{n} \& p \Vdash_{\mathbb{P}}\right.$ $\check{\alpha} \in \dot{T}\}$. Then, for every $q \leq r^{\star}$, we have $q \Vdash_{\mathbb{P}_{\ell(q)}}$ " $\dot{T}_{\ell(q)}$ is nonstationary."

Proof Towards a contradiction, suppose that there exists $q \leq r^{\star}$ such that $q \psi_{\mathbb{P}_{\ell(q)}}$ " $\dot{T}_{\ell(q)}$ is nonstationary." Consequently, we may pick $p \leq \leq^{0} q$ such that $p \Vdash_{\mathbb{P}_{n}}$ " $\dot{T}_{n}$ is stationary," for $n:=\ell(q)$. Let $G_{n}$ be $\mathbb{P}_{n}$-generic with $p \in G_{n}$. As $V\left[G_{n}\right] \vDash$ $\operatorname{Refl}\left(1, E_{\omega}^{\mu}, E_{<\kappa_{n}}^{\mu}\right)$, let us fix $p^{\prime} \leq^{0} p$ in $G_{n}$, and some $\delta \in E_{<\kappa_{n}}^{\mu}$ of uncountable cofinality such that $p^{\prime} \Vdash \mathbb{P}_{n}$ " $\dot{T}_{n} \cap \delta$ is stationary." As $\mathbb{P}_{n}$ is $\kappa_{n}$-closed, $\delta \in \Gamma$. In $V$, pick a club $C \subseteq \delta$ of order type $\operatorname{cf}(\delta)$. Note that $|C|<\kappa_{n}$. Then, $p^{\prime} \Vdash \mathbb{P}_{n}$ “ $\dot{T}_{n} \cap C$ is stationary in $\delta$." Working for a moment in $V\left[G_{n}\right]$, write $A:=C \cap\left(\dot{T}_{n}\right)_{G_{n}}$. Since $\mathbb{P}_{n}$ is $\kappa_{n}$-closed, we may find $r \in P_{n}$ extending $p^{\prime}$ that decides $A$ to be some ground model stationary subset $B$ of $\delta$. Namely,

$$
r \Vdash \mathbb{P}_{n} \text { “的 } \cap \delta \text { contains the stationary set } \check{B} \text {.” }
$$

By definition of the name $\dot{T}_{n}$, we have that $r \Vdash_{\mathbb{P}} \check{B} \subseteq \dot{T} \cap \delta$. Finally, as otp $(B)<\kappa$, we infer from Lemma 2.10(1) that $B$ remains stationary in any forcing extension by $\mathbb{P}$. So, $r \leq p^{\prime} \leq p \leq q \leq r^{\star}$, and $r \Vdash_{\mathbb{P}}$ " $\dot{T} \cap \delta$ is stationary, " contradicting the fact that $r^{\star}$ forces $\dot{T}$ to not reflect in $\Gamma$.

Suppose $r^{\star} \in P$ forces that $\dot{T}$ is a $\mathbb{P}$-name for a stationary subset $T$ of $\left(E_{\omega}^{\mu}\right)^{V}$ that does not reflect in $\Gamma$. We shall devise a $\Sigma$-Prikry notion of forcing $\left(\mathbb{A}, \ell_{\mathbb{A}}, c_{\mathbb{A}}\right)$ such that $\mathbb{A}=\mathbb{A}(\mathbb{P}, \dot{T})$ projects to $\mathbb{P}$ and kills the stationarity of $T$. Moreover, $\left(\mathbb{A}, \ell_{\mathbb{A}}, c_{\mathbb{A}}\right)$ will admit a forking projection to $(\mathbb{P}, \ell, c)$ with the mixing property.

Here goes. For all $n<\omega$, write $\dot{T}_{n}:=\left\{(\check{\alpha}, p) \mid(\alpha, p) \in E_{\omega}^{\mu} \times P_{n} \quad \& p \Vdash_{\mathbb{P}} \check{\alpha} \in\right.$ $\dot{T}\}$. Let $I:=\omega \backslash \ell\left(r^{\star}\right)$. By Lemma 6.1, for all $q \leq r^{\star}$ with $\ell(q) \in I, q \Vdash_{\mathbb{P}_{\ell(q)}}$ " $\dot{T}_{\ell(q)}$ is nonstationary." Thus, for each $n \in I$, we may pick a $\mathbb{P}_{n}$-name $\dot{C}_{n}$ for a club subset of $\mu$ such that, for all $q \leq r^{\star}$ with $\ell(q)=n$,

$$
q \Vdash \mathbb{P}_{n} \dot{T}_{n} \cap \dot{C}_{n}=\varnothing .
$$

\footnotetext{
${ }^{19}$ The easy proof may be found in [18].

${ }^{20}$ In particular, $\kappa_{n}>\aleph_{1}$ in $V^{\mathbb{P}_{n}}$.
} 
Consider the binary relation $R$ as defined in Lemma 5.6 (page 1222) with respect to $\left\langle\dot{C}_{n} \mid n \in I\right\rangle$. A moment reflection makes it clear that, for all $(\alpha, q) \in R, q \Vdash \mathbb{P} \check{\alpha} \notin \dot{T}$.

Definition 6.2 Suppose $p \in P$. A labeled $p$-tree is a function $S: W(p) \rightarrow[\mu]^{<\mu}$ such that for all $q \in W(p)$ :

(1) $S(q)$ is a closed bounded subset of $\mu$;

(2) $S\left(q^{\prime}\right) \supseteq S(q)$ whenever $q^{\prime} \leq q$;

(3) $q \Vdash_{\mathbb{P}} S(q) \cap \dot{T}=\varnothing$;

(4) for all $q^{\prime} \leq q$ in $W(p)$, either $S\left(q^{\prime}\right)=\varnothing$ or $\left(\max \left(S\left(q^{\prime}\right)\right), q\right) \in R$.

Definition 6.3 For $p \in P$, we say that $\vec{S}=\left\langle S_{i} \mid i \leq \alpha\right\rangle$ is a $p$-strategy iff all of the following hold:

(1) $\alpha<\mu$;

(2) $S_{i}$ is a labeled $p$-tree for all $i \leq \alpha$;

(3) for every $i<\alpha$ and $q \in W(p), S_{i}(q) \sqsubseteq S_{i+1}(q)$;

(4) for every $i<\alpha$ and a pair $q^{\prime} \leq q$ in $W(p),\left(S_{i+1}(q) \backslash S_{i}(q)\right) \sqsubseteq\left(S_{i+1}\left(q^{\prime}\right) \backslash S_{i}\left(q^{\prime}\right)\right)$;

(5) for every limit $i \leq \alpha$ and $q \in W(p), S_{i}(q)$ is the ordinal closure of $\cup_{j<i} S_{j}(q)$. In particular, $S_{0}(q)=\varnothing$ for all $q \in W(p)$.

This section centers around the following notion of forcing.

Definition 6.4 Let $\mathbb{A}(\mathbb{P}, \dot{T})$ be the notion of forcing $\mathbb{A}:=(A, \unlhd)$, where:

(1) $(p, \vec{S}) \in A$ iff $p \in P$, and $\vec{S}$ is either the empty sequence, or a $p$-strategy;

(2) $\left(p^{\prime}, \overrightarrow{S^{\prime}}\right) \unlhd(p, \vec{S})$ iff:

(a) $p^{\prime} \leq p$

(b) $\operatorname{dom}\left(\vec{S}^{\prime}\right) \geq \operatorname{dom}(\vec{S})$;

(c) $S_{i}^{\prime}(q)=S_{i}(w(p, q))$ for all $i \in \operatorname{dom}(\vec{S})$ and $q \in W\left(p^{\prime}\right)$.

For all $p \in P$, denote $\lceil p\rceil^{\mathbb{A}}:=(p, \varnothing)$.

Remark 6.5 The relation $\unlhd$ is well-defined as $w(p, q) \in W(p)$, the domain of the p-labeled trees $S_{i}$.

It is easy to see that $1_{\mathbb{A}}=\left\lceil 1_{\mathbb{P}}\right\rceil^{\mathbb{A}}$.

Lemma 6.6 For every $v \geq \mu$, if $\mathbb{P}$ is a subset of $H_{v}$, then so is $\mathbb{A}$.

Proof Suppose $\mathbb{P} \subseteq H_{v}$ for a given $v \geq \mu$. To prove that $\mathbb{A} \subseteq H_{v}$, it suffices to show that $A \subseteq H_{v}$. Now, each element of $A$ is a pair $(p, \vec{S})$, with $p \in P \subseteq H_{v}$ and $\vec{S} \in$ ${ }^{<\mu}\left({ }^{W(p)}[\mu]^{<\mu}\right)$, so, as $v \geq \mu$, it suffices to show that ${ }^{W(p)}[\mu]^{<\mu} \subseteq H_{v}$. Any element of $W(p)[\mu]^{<\mu}$ is a subset of $W(p) \times[\mu]^{<\mu}$ of size $|W(p)|$ and, in particular, a subset of $H_{v} \times H_{\mu}$ of size $<\mu$ because of Definition 2.3(5), so that it is indeed an element of $H_{v}$.

Lemma 6.7 Suppose $(p, \vec{S}) \in A$, where $p$ is compatible with $r^{\star}$. For every $\varepsilon<\mu$, there exist $\alpha>\varepsilon$ and $(q, \vec{T}) \unlhd(p, \vec{S})$ such that, for all $r \in W(q), \operatorname{dom}(\vec{T})=\alpha+1$ and $\max \left(T_{\alpha}(r)\right)=\alpha$.

Proof Fix $p^{\prime} \leq p, r^{\star}$. Define a $p^{\prime}$-strategy $\vec{S}^{\prime}$ with $\operatorname{dom}(\vec{S})=\operatorname{dom}\left(\vec{S}^{\prime}\right)$ using Clause (2c) of Definition 6.4, $\left(p^{\prime}, \vec{S}^{\prime}\right) \unlhd(p, \vec{S})$. Next, let $\varepsilon<\mu$ be arbitrary. Since 
$(\mathbb{P}, \ell, c)$ is $\Sigma$-Prikry, we infer from Definition 2.3(5) that $\left|W\left(p^{\prime}\right)\right|<\mu$. Thus, by possibly extending $\varepsilon$, we may assume that $S_{i}^{\prime}(q) \subseteq \varepsilon$, for all $q \in W\left(p^{\prime}\right)$ and $i \in \operatorname{dom}\left(\vec{S}^{\prime}\right)$.

Assume for a moment that $\vec{S}^{\prime} \neq \varnothing$ and write $\delta+1:=\operatorname{dom}\left(\vec{S}^{\prime}\right)$. As $p^{\prime} \leq r^{\star}$, by the very same proof of Claim 5.6.2(1), we may fix $(\alpha, q) \in R$ with $\alpha>\delta+\varepsilon$ and $q \leq p^{\prime}$. Define $\vec{T}=\left\langle T_{i}: W(q) \rightarrow[\mu]^{<\mu} \mid i \leq \alpha\right\rangle$ by letting for all $r \in W(q)$ and $i \in \operatorname{dom}(\vec{T})$ :

$$
T_{i}(r):= \begin{cases}S_{i}^{\prime}\left(w\left(p^{\prime}, r\right)\right), & \text { if } i \leq \delta ; \\ S_{\delta}^{\prime}\left(w\left(p^{\prime}, r\right)\right) \cup\{\alpha\}, & \text { otherwise. }\end{cases}
$$

It is easy to see that $T_{i}$ is a labeled $q$-tree for each $i \leq \alpha$. By Definitions 6.3 and 6.4, we also have that $(q, \vec{T})$ is a condition in $\mathbb{A}$ and $(q, \vec{T}) \unlhd\left(p^{\prime}, \vec{S}^{\prime}\right) \unlhd(p, \vec{S})$. Altogether, $\alpha$ and $(q, \vec{T})$ are as desired.

In case $\vec{S}=\varnothing$, arguing as before we may find $(\alpha, q) \in R$ with $\alpha>\varepsilon$ and $q \leq p^{\prime}$. Define $\vec{T}=\left\langle T_{i}: W(q) \rightarrow[\mu]^{<\mu} \mid i \leq \alpha\right\rangle$ by letting for all $r \in W(q)$ and $i \in \operatorname{dom}(\vec{T})$ :

$$
T_{i}(r):= \begin{cases}\varnothing, & \text { if } i=0 \\ \{\alpha\}, & \text { otherwise }\end{cases}
$$

It is clear that $\vec{T}$ is a $q$-strategy and that $(q, \vec{T})$ is as desired.

Theorem $6.8\left(r^{\star}, \varnothing\right) \Vdash_{\mathbb{A}}$ “ $\dot{T}$ is nonstationary."

Proof Let $G$ be $\mathbb{A}$-generic over $V$, with $\left(r^{\star}, \varnothing\right) \in G$. Work in $V[G]$. Let $\bar{G}$ be the induced generic for $\mathbb{P}$ via $\pi$, so that $r^{\star} \in \bar{G}$.

For all $a=(p, \vec{S})$ in $G$ and $i \in \operatorname{dom}(\vec{S})$, write $d_{a}^{i}:=\bigcup\left\{S_{i}(q) \mid q \in \bar{G} \cap W(p)\right\}$. Then, let

$$
d_{a}:= \begin{cases}d_{a}^{\max (\operatorname{dom}(\vec{S}))}, & \text { if } \vec{S} \neq \varnothing ; \\ \varnothing, & \text { otherwise }\end{cases}
$$

Claim 6.8.1 Suppose that $a=(p, \vec{S})$ is an element of $G$.

In $V[\bar{G}]$, for all $i \in \operatorname{dom}(\vec{S})$, the ordinal closure $\operatorname{cl}\left(d_{a}^{i}\right)$ of $d_{a}^{i}$ is disjoint from $T$.

Proof Work in $V[\bar{G}]$. By Lemma 2.8(1), for all $n<\omega$, there exists a unique element in $\bar{G} \cap W_{n}(p)$, which we shall denote by $p_{n}$. By Lemma 2.8(2), it follows that $\left\langle p_{n} \mid n<\omega\right\rangle$ is $\leq$-decreasing and then, by Definition 6.2, for each $i \in \operatorname{dom}(\vec{S}),\left\langle S_{i}\left(p_{n}\right) \mid n<\omega\right\rangle$ is a weakly $\subseteq$-increasing (though, not $\subseteq$-increasing) sequence of closed sets that converges to $d_{a}^{i}$.

We now argue by induction on $i \in \operatorname{dom}(\vec{S})$. The base case is trivial, since $d_{a}^{0}=\varnothing$.

Next, suppose that the claim holds for a given $i<\max (\operatorname{dom}(\vec{S}))$, and let us prove it for $i+1$. Let $\delta \in \operatorname{cl}\left(d_{a}^{i+1}\right) \backslash \mathrm{cl}\left(d_{a}^{i}\right)$ be arbitrary. We have to verify that $\delta \notin T$. By Clauses (3) and (4) of Definition 6.2, we may assume that $\delta \in \operatorname{cl}\left(d_{a}^{i+1}\right) \backslash d_{a}^{i+1}$. In particular, as $d_{a}^{i+1}$ is the countable union of closed sets, we have $\operatorname{cf}(\delta)=\omega$.

Subclaim 6.8.1.1 There exists a sequence $\left\langle\delta_{n} \mid n \in N\right\rangle$ of ordinals in $\delta$ such that:

- $N \in[\omega]^{\omega}$;

- $\sup _{n \in N} \delta_{n}=\delta$;

- for every $n \in N, n=\min \left\{\bar{n}<\omega \mid \delta_{n} \in S_{i+1}\left(p_{\bar{n}}\right) \backslash S_{i}\left(p_{\bar{n}}\right)\right\}$. 
Proof Since $\delta \in \operatorname{cl}\left(d_{a}^{i+1}\right) \backslash\left(\operatorname{cl}\left(d_{a}^{i}\right) \cup d_{a}^{i+1}\right)$ and $\operatorname{cf}(\delta)=\omega$, we may find a strictly increasing sequence $\left\langle\delta^{m} \mid m<\omega\right\rangle$ of ordinals in $d_{a}^{i+1} \backslash d_{a}^{i}$ such that $\sup _{m<\omega} \delta^{m}=\delta$. For each $m<\omega$, let $n_{m}<\omega$ be the least such that $\delta^{m} \in S_{i+1}\left(p_{n_{m}}\right) \backslash S_{i}\left(p_{n_{m}}\right)$. Since $S_{i+1}\left(p_{n}\right)$ is closed for every $n<\omega$, we get that $m \mapsto n_{m}$ is finite-to-one, so that $N:=\left\{n_{m} \mid\right.$ $m<\omega\}$ is infinite. For each $n \in N$, set $m(n):=\min \left\{m<\omega \mid n=n_{m}\right\}$ and $\delta_{n}:=\delta^{m(n)}$. Evidently,

$$
\begin{gathered}
\min \left\{\bar{n}<\omega \mid \delta_{n} \in S_{i+1}\left(p_{\bar{n}}\right) \backslash S_{i}\left(p_{\bar{n}}\right)\right\}= \\
\min \left\{\bar{n}<\omega \mid \delta^{m(n)} \in S_{i+1}\left(p_{\bar{n}}\right) \backslash S_{i}\left(p_{\bar{n}}\right)\right\}= \\
n_{m(n)}=n .
\end{gathered}
$$

In particular, $\langle m(n) \mid n \in N\rangle$ is injective, and $\sup _{n \in N} \delta_{n}=\delta$.

Let $\left\langle\delta_{n} \mid n \in N\right\rangle$ be given by the subclaim. By Definition 6.3(3), for all $n<m<\omega$, we have $\left(S_{i+1}\left(p_{n}\right) \backslash S_{i}\left(p_{n}\right)\right) \sqsubseteq\left(S_{i+1}\left(p_{m}\right) \backslash S_{i}\left(p_{m}\right)\right)$, and hence $\delta=\sup _{n \in N} \sup \left(S_{i+1}\left(p_{n}\right) \backslash S_{i}\left(p_{n}\right)\right)$. Recalling that $S_{i}\left(p_{n}\right) \sqsubseteq S_{i+1}\left(p_{n}\right)$ for all $n<\omega$, we conclude that

$$
\delta=\sup _{n \in N} \max \left(S_{i+1}\left(p_{n}\right)\right) .
$$

By Definition 6.2(4), we have $\left(\max \left(S_{i+1}\left(p_{m}\right)\right), p_{n}\right) \in R$ for all $n \in N$ and $m \geq n$. So, since, for each $m \in I, \dot{C}_{m}$ is a $\mathbb{P}_{m}$-name for a club, we infer that $\left(\delta, p_{n}\right) \in R$ for all $n \in N$. Recalling the definition of $R$ and the fact that $I=\omega \backslash \ell\left(r^{\star}\right)$, we infer that, for every $n \geq \min (N), p_{n} \leq r^{\star}$, and

$$
p_{n} \Vdash \mathbb{P}_{n} \check{\delta} \in \dot{C}_{n} .
$$

Now, for every $n \geq \min (N)$, by the very choice of $\dot{C}_{n}$ and since $p_{n} \leq r^{\star}, p_{n} \Vdash \mathbb{P}_{n} \dot{T}_{n} \cap$ $\dot{C}_{n}=\varnothing$. Altogether, for a tail of $n<\omega$,

$$
p_{n} \Vdash \mathbb{P}_{n} \check{\delta} \notin \dot{T}_{n} .
$$

It thus follows from the definition of $\left\langle\dot{T}_{n} \mid n<\omega\right\rangle$ and the fact that $\left\{p_{n} \mid n<\omega\right\} \subseteq \bar{G}$, that $\delta \notin T$.

Finally, suppose $i \in \operatorname{acc}^{+}(\operatorname{dom}(\vec{S}))$, and that the claim holds below $i$. Let $\delta \in$ $\operatorname{cl}\left(d_{a}^{i}\right) \backslash d_{a}^{i}$ be arbitrary. By the previous analysis, it is clear that we may pick $N \in[\omega]^{\omega}$ and an increasing sequence of ordinals $\left\langle\delta_{n} \mid n \in N\right\rangle$ that converges to $\delta$, such that $\delta_{n} \in S_{i}\left(p_{n}\right)$ for all $n \in N$. By the last clause of Definition 6.3, for each $n \in N$, we may let $j_{n}<i$ be the least for which there exists $\delta_{n}^{\prime} \in S_{j_{n}+1}\left(p_{n}\right)$ with $\delta_{n} \geq \delta_{n}^{\prime}>\sup \left\{\delta_{m} \mid\right.$ $m \in N \cap n\}$.

If $\sup _{n \in N} j_{n}<i$, then by the induction hypothesis, $\delta \notin T$, and we are done. Suppose that $\sup _{n \in N} j_{n}=i$. By thinning $N$ out, we may assume that $n \mapsto j_{n}$ is strictly increasing over $N$. In particular, for all $m<n$ both from $N$, we have $\delta_{m}^{\prime} \in S_{j_{m}+1}\left(p_{m}\right) \subseteq$ $S_{j_{n}}\left(p_{m}\right) \subseteq S_{j_{n}}\left(p_{n}\right) \subseteq S_{j_{n}+1}\left(p_{n}\right)$, so that $\delta_{m}^{\prime} \leq \max \left(S_{j_{n}}\left(p_{n}\right)\right) \leq \delta_{n}^{\prime}$. Altogether, $\delta=$ $\sup _{n \in N} \max \left(S_{j_{n}}\left(p_{n}\right)\right)$. By Definition 6.2(4), we have $\left(\max \left(S_{j_{n}}\left(p_{m}\right)\right), p_{n}\right) \in R$ whenever $n \in N$ and $m \in \omega \backslash n$. Thus, as in the successor case, we have $\left(\delta, p_{n}\right) \in R$ for all $n \in N$, and hence $\delta \notin T$. 
By appealing to Lemma 6.7, we now fix a sequence $\left\langle a_{\alpha} \mid \alpha<\mu\right\rangle$ of conditions in $G$ such that, for all $\alpha<\mu$, letting $(p, \vec{S}):=a_{\alpha}$, we have $\operatorname{dom}(\vec{S})=\alpha+1$. Denote $D_{\alpha}:=$ $\mathrm{cl}\left(d_{a_{\alpha}}\right)$. By the preceding claim and regularity of $\mu$ we infer: ${ }^{21}$

Claim 6.8.2 For every $\alpha<\mu, D_{\alpha}$ is a closed bounded subset of $\mu$, disjoint from $T$.

Claim 6.8.3 For every $\alpha<\mu$ and $a^{\prime}=\left(p^{\prime}, \vec{S}^{\prime}\right)$ in $G$ with $\operatorname{dom}\left(\vec{S}^{\prime}\right)=\alpha+1, d_{a^{\prime}}=d_{a_{\alpha}}$.

Proof Denote $a_{\alpha}=(p, \vec{S})$. As $a_{\alpha}$ and $a^{\prime}$ are in $G$, we may pick $(r, \vec{T})$ that extends both. In particular, $r \leq p, p^{\prime}$, and, for all $q \in W(r), S_{\alpha}(w(p, q))=T_{\alpha}(q)=$ $S_{\alpha}^{\prime}\left(w\left(p^{\prime}, q\right)\right)$. Let $m:=\ell(r)-\ell(p)$. Then, for all $k<\omega, q \in W_{k}(r) \cap G$ iff $w(p, q) \in$ $W_{m+k}(p) \cap G$. Note that these sets are singletons. Then

$$
d_{a_{\alpha}}=\bigcup\left\{S_{\alpha}(q) \mid q \in \bar{G} \cap W_{\geq m}(p)\right\}=\bigcup\left\{T_{\alpha}(q) \mid q \in \bar{G} \cap W(r)\right\} .
$$

Similarly, we have that $d_{a^{\prime}}=\bigcup\left\{T_{\alpha}(q) \mid q \in \bar{G} \cap W(r)\right\}$, and so $d_{a_{\alpha}}=d_{a^{\prime}}$.

Claim 6.8.4 For every $\alpha<\beta<\mu, D_{\alpha} \sqsubseteq D_{\beta}$.

Proof Let $\alpha<\beta<\mu$. It suffices to show that $d_{a_{\alpha}} \sqsubseteq d_{a_{\beta}}$. Let $(p, \vec{S}):=a_{\beta}$ and set $a:=$ $(p, \vec{S} \uparrow(\alpha+1))$. As $a_{\beta} \unlhd a$, we infer that $a \in G$. Thus, the preceding claim yields $d_{a}=$ $d_{a_{\alpha}}$. Let $\left\langle p_{n} \mid n<\omega\right\rangle$ be the decreasing sequence of conditions such that $p_{n}$ is unique element of $\bar{G} \cap W_{n}(p)$. Then:

- $d_{a_{\alpha}}=\bigcup\left\{S_{\alpha}\left(p_{n}\right) \mid n<\omega\right\}$, and

- $d_{a_{\beta}}=\bigcup\left\{S_{\beta}\left(p_{n}\right) \mid n<\omega\right\}$.

Note that by Clauses (3) and (5) of Definition 6.3, for all $n<\omega, S_{\alpha}\left(p_{n}\right) \sqsubseteq S_{\beta}\left(p_{n}\right)$. Now, let $\gamma<\mu$ be arbitrary. We consider two cases:

- If $\gamma \in d_{a_{\alpha}}$, then we may find $n<\omega$ such that $\gamma \in S_{\alpha}\left(p_{n}\right)$, and as $S_{\alpha}\left(p_{n}\right) \sqsubseteq S_{\beta}\left(p_{n}\right)$, we infer that $\gamma \in d_{a_{\beta}}$.

- If $\gamma \in d_{a_{\beta}} \backslash d_{a_{\alpha}}$, then we first find $n<\omega$ such that $\gamma \in S_{\beta}\left(p_{n}\right)$. In particular, $\gamma \in S_{\beta}\left(p_{n}\right) \backslash S_{\alpha}\left(p_{n}\right)$, and as $S_{\alpha}\left(p_{n}\right) \sqsubseteq S_{\beta}\left(p_{n}\right)$, this means that $\gamma \geq \sup \left(S_{\alpha}\left(p_{n}\right)\right)$. By Definition 6.2(2), for all $m \geq n, S_{\beta}\left(p_{n}\right) \subseteq S_{\beta}\left(p_{m}\right)$, and so it likewise follows that, for all $m \geq n, \gamma \geq \sup \left(S_{\alpha}\left(p_{m}\right)\right)$. By Definition 6.2(2), for all $m<n, S_{\alpha}\left(p_{m}\right) \subseteq S_{\alpha}\left(p_{n}\right)$, and so $\gamma \geq \sup \left(S_{\alpha}\left(p_{n}\right)\right) \geq \sup \left(S_{\alpha}\left(p_{m}\right)\right)$. Altogether, $\gamma \geq \sup \left(d_{a_{\alpha}}\right)$.

Claim 6.8.5 For every $\varepsilon<\mu$, there exists $\alpha<\mu$ such that $\max \left(D_{\alpha}\right)>\varepsilon$.

Proof By Lemma 6.7, we may find $(q, \vec{T})$ in $G$ and $\alpha>\varepsilon$ such that, for all $r \in W(q)$, $\operatorname{dom}(\vec{T})=\alpha+1$ and $\max \left(T_{\alpha}(r)\right)=\alpha$. By Claim 6.8.3, then, $\max \left(D_{\alpha}\right)=\alpha>\varepsilon$.

Put $D:=\bigcup\left\{D_{\alpha} \mid \alpha<\mu\right\}$. By Claims 6.8.2 and 6.8.4,D is closed subset of $\mu$, disjoint from $T$. By Claim 6.8.5, $D$ is unbounded. So $T$ is nonstationary in $V[G]$.

Definition 6.9 Let $\ell_{\mathbb{A}}:=\ell \circ \pi$. Denote $A_{n}:=\left\{a \in A \mid \ell_{\mathbb{A}}(a)=n\right\}, A_{n}^{a}:=\left\{a^{\prime} \in A \mid\right.$ $\left.a^{\prime} \unlhd a, \ell_{\mathbb{A}}\left(a^{\prime}\right)=\ell_{\mathbb{A}}(a)+n\right\}$, and $\mathbb{A}_{n}:=\left(A_{n} \cup\left\{1_{\mathbb{A}}\right\}, \unlhd\right)$.

Definition 6.10 Define $c_{\mathbb{A}}: A \rightarrow H_{\mu}$ by letting, for all $(p, \vec{S}) \in A$,

$$
c_{\mathbb{A}}(p, \vec{S}):=\left(c(p),\left\{\left(i, c(q), S_{i}(q)\right) \mid i \in \operatorname{dom}(\vec{S}), q \in W(p)\right\}\right) .
$$

\footnotetext{
${ }^{21}$ See Corollary 4.13.
} 
The rest of this section is devoted to verifying that $\left(\mathbb{A}, \ell_{\mathbb{A}}, c_{\mathbb{A}}\right)$ is a $\sum$-Prikry forcing that admits a forking projection to $(\mathbb{P}, \ell, c)$.

Definition 6.11 (Projection and forking)

- Define $\pi: A \rightarrow P$ by stipulating $\pi(p, \vec{S}):=p$.

- Given $a=(p, \vec{S})$ in $A$, define $h(a): \mathbb{P} \downarrow p \rightarrow A$ by letting for each $p^{\prime} \leq p$, $\pitchfork(a)\left(p^{\prime}\right):=\left(p^{\prime}, \vec{S}^{\prime}\right)$, where $\vec{S}^{\prime}$ is the sequence $\left\langle S_{i}^{\prime}: W\left(p^{\prime}\right) \rightarrow[\mu]^{<\mu} \mid i<\operatorname{dom}(\vec{S})\right\rangle$ to satisfy:

$$
S_{i}^{\prime}(q):=S_{i}(w(p, q)) \text { for all } i \in \operatorname{dom}\left(\vec{S}^{\prime}\right) \text { and } q \in W\left(p^{\prime}\right) .
$$

Lemma 6.12 Let $a \in A$ and $p^{\prime} \leq \pi(a)$. Then $\pitchfork(a)\left(p^{\prime}\right) \in A$ and $\downarrow(a)\left(p^{\prime}\right) \unlhd a$, so that $\downarrow(a)$ is a well-defined function from $\mathbb{P} \downarrow \pi(a)$ to $\mathbb{A} \downarrow$ a.

Proof Set $a:=(p, \vec{S})$. If $\vec{S}=\varnothing$, then $\pitchfork(a)\left(p^{\prime}\right)=\left\lceil p^{\prime}\right\rceil^{\mathbb{A}}$, and we are done.

Next, suppose that $\operatorname{dom}(\vec{S})=\alpha+1$. Let $\left(p^{\prime}, \vec{S}^{\prime}\right):=\hbar(a)\left(p^{\prime}\right)$. Let $i \leq \alpha$ and we shall verify that $S_{i}^{\prime}$ is a $p^{\prime}$-labeled tree. To this end, let $q^{\prime} \leq q$ be arbitrary pair of elements of $W\left(p^{\prime}\right)$.

- By Definition 2.3(6), we have $w\left(p, q^{\prime}\right) \leq w(p, q)$, so that $S_{i}^{\prime}\left(q^{\prime}\right)=S_{i}\left(w\left(p, q^{\prime}\right)\right) \supseteq$ $S_{i}(w(p, q))=S_{i}^{\prime}(q)$.

- As $q \leq w(p, q), \quad w(p, q) \Vdash_{\mathbb{P}} S_{i}(w(p, q)) \cap \dot{T}=\varnothing$, so that, since $S_{i}^{\prime}(q)=$ $S_{i}(w(p, q))$, we clearly have $q \Vdash_{\mathbb{P}} S_{i}^{\prime}(q) \cap \dot{T}=\varnothing$.

- To avoid trivialities, suppose that $S_{i}^{\prime}\left(q^{\prime}\right) \neq \varnothing$. Write $\gamma:=\max \left(S_{i}(w(p, q))\right.$. As $(\gamma, w(p, q)) \in R$ and $q \leq w(p, q)$, we clearly have $(\gamma, q) \in R$. Recalling that $\max \left(S_{i}^{\prime}(q)\right)=\gamma$, we are done.

To prove that $\left(p^{\prime}, \vec{S}^{\prime}\right)$ is a condition in $A$ it remains to argue that $\vec{S}^{\prime}$ fulfills the requirements described in Clauses (3) and (5) of Definition 6.3 but this already follows from the definition of $\vec{S}^{\prime}$ and the fact that $\vec{S}$ is a $p$-strategy. Finally $h(a)\left(p^{\prime}\right)=$ $\left(p^{\prime}, \vec{S}^{\prime}\right) \unlhd(p, \vec{S})=a$ by the very choice of $p^{\prime}$ and by Definition 6.11.

Let us now check that the pair of functions $(h, \pi)$ of Definition 6.11 is a forking projection from $\left(\mathbb{A}, \ell_{\mathbb{A}}, c_{\mathbb{A}}\right)$ to $(\mathbb{P}, \ell, c)$. We prove this by going over the clauses of Definition 4.1.

Lemma 6.13 (1) $\pi$ is a projection from $\mathbb{A}$ onto $\mathbb{P}$, and $\ell_{\mathbb{A}}=\ell \circ \pi$;

(2) for all $a \in A, \pitchfork(a)$ is an order-preserving function from $(\mathbb{P} \downarrow \pi(a), \leq)$ to $(\mathbb{A} \downarrow a, \unlhd)$;

(3) for all $p \in P,(p, \varnothing)$ is the greatest element of $\{a \in A \mid \pi(a)=p\}$;

(4) for all $n, m<\omega$ and $b \unlhd^{n+m} a, m(a, b)$ exists and satisfies:

$$
m(a, b)=h(a)(m(\pi(a), \pi(b))) ;
$$

(5) for all $a \in A$ and $p^{\prime} \leq \pi(a), \pi\left(\pitchfork(a)\left(p^{\prime}\right)\right)=p^{\prime}$;

(6) for all $a \in A$ and $p^{\prime} \leq \pi(a), a=(\pi(a), \varnothing)$ iff $\pitchfork(a)\left(p^{\prime}\right)=\left(p^{\prime}, \varnothing\right)$;

(7) for all $a \in A, a^{\prime} \unlhd a$ and $r \leq \pi\left(a^{\prime}\right)$, $\pitchfork\left(a^{\prime}\right)(r) \unlhd \pitchfork(a)(r)$;

(8) for all $a, a^{\prime} \in A$, if $c_{\mathbb{A}}(a)=c_{\mathbb{A}}\left(a^{\prime}\right)$, then $c(\pi(a))=c\left(\pi\left(a^{\prime}\right)\right)$ and $\pitchfork(a)(r)=$ $\pitchfork\left(a^{\prime}\right)(r)$ for every $r \leq \pi(a), \pi\left(a^{\prime}\right)$.

Proof (1) The equality between the lengths comes from Definition 6.9 so let us concentrate on proving that $\pi$ forms a projection. Clearly, $\pi\left(1_{\mathbb{A}}\right)=1_{\mathbb{P}}$. By 
Definition 6.4, for all $a^{\prime} \unlhd a$ in $A$, we have $\pi\left(a^{\prime}\right) \leq \pi(a)$. Finally, suppose that $a \in$ $A$ and $p^{\prime} \leq \pi(a)$, and let us find $a^{\prime} \unlhd a$ such that $\pi\left(a^{\prime}\right) \leq p^{\prime}$. Put $a^{\prime}:=\pitchfork(a)\left(p^{\prime}\right)$. Then it is not hard to check that $a^{\prime} \unlhd a$ and $\pi\left(h(a)\left(p^{\prime}\right)\right)=p^{\prime}$, so we are done.

(2) Let $a=(p, \vec{S})$ be an arbitrary element of $A$. By Lemma 6.12, $\pitchfork(a)$ is a function from $\mathbb{P} \downarrow \pi(a)$ to $\mathbb{A} \downarrow a$. To see that it is order-preserving, fix $r \leq q$ below $\pi(a)$. By Definition 6.11, $\pitchfork(a)(r)=(r, \vec{R})$ and $\pitchfork(a)(q)=(q, \vec{Q})$, where $\vec{R}$ and $\vec{Q}$ are as described in Definition 6.11(*). In particular, $\operatorname{dom}(\vec{R})=\operatorname{dom}(\vec{S})=$ $\operatorname{dom}(\vec{Q})$. So, to establish that $\pitchfork(a)(r) \unlhd \pitchfork(a)(q)$, it suffices to verify Clause (2c) of Definition 6.4. Let $i \in \operatorname{dom}(\vec{R})$ and $r^{\prime} \in W(r)$ be arbitrary and notice that $\left.{ }^{*}\right)$ implies $R_{i}\left(r^{\prime}\right)=S_{i}\left(w\left(p, r^{\prime}\right)\right)$. Since $r \leq q$, hence $w\left(q, r^{\prime}\right) \in W(q)$, again by $\left.{ }^{*}\right), Q_{i}\left(w\left(q, r^{\prime}\right)\right)=S_{i}\left(w\left(p, w\left(q, r^{\prime}\right)\right)\right)$. Using Lemma 2.9, it is the case that $Q_{i}\left(w\left(q, r^{\prime}\right)\right)=S_{i}\left(w\left(p, r^{\prime}\right)\right)$, hence $R_{i}\left(r^{\prime}\right)=Q_{i}\left(w\left(q, r^{\prime}\right)\right)$.

(3) This is easy to see.

(4) Write $a=(p, \vec{S})$ and $b=(\bar{p}, \vec{T})$. Appealing to Definition 2.3(4), set $p^{\prime}:=m(p, \bar{p})$, so that $\bar{p} \leq^{m} p^{\prime} \leq^{n} p$. Now, let $a^{\prime}:=\pitchfork(a)\left(p^{\prime}\right)$. By Definition 6.11, $a^{\prime}$ takes the form $\left(p^{\prime}, \vec{S}^{\prime}\right)$, where $\operatorname{dom}\left(\vec{S}^{\prime}\right)=\operatorname{dom}(\vec{S})$, and $S_{i}^{\prime}(q):=S_{i}(w(p, q))$, for all $i \in$ $\operatorname{dom}\left(\vec{S}^{\prime}\right)$ and $q \in W\left(p^{\prime}\right)$. Observe that if we prove $a^{\prime}=m(a, b)$, i.e., that $a^{\prime}$ is the greatest element of $\left\{c \in A_{n}^{a} \mid c \in A_{m}^{b}\right\}$, we will be done with both assertions.

Claim 6.13.1 $a^{\prime}$ belongs to $\left\{c \in A_{n}^{a} \mid c \in A_{m}^{b}\right\}$.

Proof By Clauses (1) and (2) together with Clause (5) below, $a^{\prime}$ is an element of $A_{n}^{a}$, so it suffices to show that $b \unlhd a^{\prime}$.

We already know that $\bar{p} \leq^{m} p^{\prime}$ and $\operatorname{dom}(\vec{T}) \geq \operatorname{dom}(\vec{S})=\operatorname{dom}\left(\vec{S}^{\prime}\right)$, thus, by virtue of Definition 6.4 , we are left with verifying that $T_{i}(q)=S_{i}^{\prime}\left(w\left(p^{\prime}, q\right)\right)$ for all $i \in \operatorname{dom}\left(\vec{S}^{\prime}\right)$ and $q \in W(\bar{p})$.

Let $i$ and $q$ be as above. As $b \unlhd a$, we infer that $T_{i}(q)=S_{i}(w(p, q))$. By definition of $S_{i}^{\prime}$ and Lemma 2.9, $S_{i}^{\prime}\left(w\left(p^{\prime}, q\right)\right)=S_{i}\left(w\left(p, w\left(p^{\prime}, q\right)\right)=S_{i}(w(p, q))\right.$, so that, altogether, $T_{i}(q)=S_{i}^{\prime}\left(w\left(p^{\prime}, q\right)\right)$, as desired.

Claim 6.13.2 $a^{\prime}$ is the greatest element of $\left\{c \in A_{n}^{a} \mid b \in A_{m}^{b}\right\}$.

Proof Let $c=(r, \vec{R})$ be a condition with $(\bar{p}, \vec{T}) \unlhd^{m}(r, \vec{R}) \unlhd^{n}(p, \vec{S})$. In particular, $\bar{p} \leq^{m} r \leq^{n} p$, so that, since $p^{\prime}=m(p, \bar{p}), r \leq^{0} p^{\prime}$.

We already know that $r \leq p^{\prime}$ and $\operatorname{dom}(\vec{R}) \geq \operatorname{dom}(\vec{S})=\operatorname{dom}\left(\vec{S}^{\prime}\right)$. Now, let $i \in \operatorname{dom}\left(\vec{S}^{\prime}\right)$ and $q \in W(r)$ be arbitrary. By definition of $S_{i}^{\prime}$ and Lemma 2.9, $S_{i}^{\prime}\left(w\left(p^{\prime}, q\right)\right)=S_{i}\left(w\left(p, w\left(p^{\prime}, q\right)\right)=S_{i}(w(p, q))\right.$. As $c \unlhd a$, the latter is equal to $R_{i}(q)$, hence $c \unlhd a^{\prime}$, as desired.

(5) This follows immediately from Definition 6.11.

(6) Suppose that $a \in A$ with $a=(\pi(a), \varnothing)$. By Definition 6.11(*), for all $p^{\prime} \leq \pi(a)$, $h(a)\left(p^{\prime}\right)=\left(p^{\prime}, \varnothing\right)$. Conversely, let $a:=(\pi(a), \vec{S})$ and suppose that $h(a)(q)=$ $(q, \varnothing)$. Again, by Definition 6.11, $\operatorname{dom}(\vec{S})=\varnothing$, and thus $a=(\pi(a), \varnothing)$, as desired.

(7) Let $a \in A, a^{\prime} \unlhd a$ and $r \leq \pi\left(a^{\prime}\right)$ be arbitrary, say $a^{\prime}=\left(p^{\prime}, \vec{S}^{\prime}\right)$ and $a=(p, \vec{S})$. By Definition 6.4, the following three hold:

- $p^{\prime} \leq p$

- $\operatorname{dom}(\vec{S}) \leq \operatorname{dom}\left(\vec{S}^{\prime}\right)$,

- $S_{i}^{\prime}(q)=S_{i}(w(p, q))$, for all $i \in \operatorname{dom}(\vec{S})$ and $q \in W\left(p^{\prime}\right)$. 
By Definition 6.11, $\pitchfork(a)(r):=\left(r, \vec{S}^{a}\right)$, where $\operatorname{dom}\left(\vec{S}^{a}\right)=\operatorname{dom}(\vec{S})$ and, for all $i<\operatorname{dom}(\vec{S})$ and $q \in W(r), S_{i}^{a}(q)=S_{i}(w(p, q))$. A similar statement is valid for $h\left(a^{\prime}\right)(r)=\left(r, \vec{S}^{a^{\prime}}\right)$. Notice that $\operatorname{dom}\left(\vec{S}^{a^{\prime}}\right) \geq \operatorname{dom}\left(\vec{S}^{a}\right)$ and that, for all $i<$ $\operatorname{dom}\left(\vec{S}^{a}\right)$ and $q \in W(r)$, Lemma 2.9 yields the following chain of equalities:

$$
S_{i}^{a^{\prime}}(q)=S_{i}^{\prime}\left(w\left(p^{\prime}, q\right)\right)=S_{i}\left(w\left(p, w\left(p^{\prime}, q\right)\right)\right)=S_{i}(w(p, q))=S_{i}^{a}(q) .
$$

Altogether, we have proved $\pitchfork_{(}\left(a^{\prime}\right)(r) \unlhd \pitchfork(a)(r)$.

(8) Let $a=(p, \vec{S})$ and $a^{\prime}=\left(p^{\prime}, \vec{S}^{\prime}\right)$ be elements of $A$ with $c_{\mathbb{A}}(a)=c_{\mathbb{A}}\left(a^{\prime}\right)$. By Definition 6.10, then, $c(\pi(a))=c\left(\pi\left(a^{\prime}\right)\right)$ and $\operatorname{dom}(\vec{S})=\operatorname{dom}\left(\vec{S}^{\prime}\right)$. Now, let $r \leq$ $\pi(a), \pi\left(a^{\prime}\right)$ be arbitrary; we shall show that $h(a)(r)=\hbar\left(a^{\prime}\right)(r)$. Recall that $\pitchfork(a)(r)=(r, \vec{T})$ and $\pitchfork\left(a^{\prime}\right)(r)=\left(r, \vec{T}^{\prime}\right)$, where $\vec{T}$ and $\vec{T}^{\prime}$ are the $r$-strategy of length $\operatorname{dom}(\vec{S})$ given by Definition $6.11\left(^{*}\right)$ with respect to $a$ and $a^{\prime}$, respectively. Therefore, it suffices to show that, for all $i \in \operatorname{dom}(\vec{S})$ and $q \in W(r), S_{i}(w(p, q))=$ $S_{i}^{\prime}\left(w\left(p^{\prime}, q\right)\right)$. Let $i \in \operatorname{dom}(\vec{S})$ and $q \in W(r)$ be arbitrary. By Lemma 2.8(4), $c \uparrow W(p)$ is injective. Since $c_{\mathbb{A}}(a)=c_{\mathbb{A}}\left(a^{\prime}\right)$, Definition 6.10 yields $c^{\text {“ }} W(p)=$

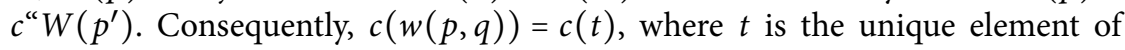
$W\left(p^{\prime}\right)$ that is compatible with $w(p, q)$ and has the same length. Thus, it is not hard to check that $t=w\left(p^{\prime}, q\right)$, hence $c(w(p, q))=c\left(w\left(p^{\prime}, q\right)\right)$. Finally, as $c_{\mathbb{A}}(a)=c_{\mathbb{A}}\left(a^{\prime}\right)$ and $c(w(p, q))=c\left(w\left(p^{\prime}, q\right)\right)$, it is the case that $S_{i}(w(p, q))=$ $S_{i}^{\prime}\left(w\left(p^{\prime}, q\right)\right)$.

Remark 6.14 Note that the above proof only uses the fact that the triple $(\mathbb{P}, \ell, c)$ is $\sum$-Prikry together with the defining properties of $\left(\mathbb{A}, \ell_{\mathbb{A}}, c_{\mathbb{A}}\right)$ (that is, Definitions 6.4, $6.9,6.10$ and 6.11). In particular, we have not relied on any clause of Definition 2.3 for $\left(\mathbb{A}, \ell_{\mathbb{A}}, c_{\mathbb{A}}\right)$, which have not yet been verified.

Lemma 6.15 Let $n<\omega$. Suppose that $D$ is a directed family of conditions in $\mathbb{A}_{n},|D|<\mu$, and for some $\bar{p}$, we have $\pi(a)=\bar{p}$ for all $a \in D$. Then $D$ admits a lower bound in $\mathbb{A}_{n}$.

Proof Since $D$ is directed, given any $a, a^{\prime} \in D$, we may pick $b \in D$ extending $a$ and $a^{\prime}$; now, as $\pi[D]=\{\bar{p}\}$, find $\vec{S}, \vec{S}^{\prime}, \vec{T}$ such that $a=(\bar{p}, \vec{S}), a^{\prime}=\left(\bar{p}, \vec{S}^{\prime}\right)$ and $b=$ $(\bar{p}, \vec{T})$, and note that, by Definition 6.4, for all $q \in W(\bar{p})$ and $i \in \operatorname{dom}(\vec{S}) \cap \operatorname{dom}\left(\vec{S}^{\prime}\right)$, $S_{i}(q)=\vec{T}_{i}(q)=S_{i}^{\prime}(q)$. It thus follows that $D$ is linearly ordered by $\unlhd$, and, for all $(\bar{p}, \vec{S}),\left(\bar{p}, \vec{S}^{\prime}\right) \in D,(\bar{p}, \vec{S}) \unlhd\left(\bar{p}, \vec{S}^{\prime}\right)$ iff $\operatorname{dom}(\vec{S}) \geq \operatorname{dom}\left(\vec{S}^{\prime}\right)$. So $(D, \unlhd)$ is orderisomorphic to $(\theta, \ni)$ for some ordinal $\theta<\mu$. In particular, if $\theta$ is a successor ordinal, then $D$ admits a lower bound. So let us assume that $\theta$ is a limit ordinal.

For every $\tau<\theta$, let $\left(\bar{p}, \vec{S}^{\tau}\right)$ denote the $\tau^{t h}$-element of $D$. Set $\alpha:=\sup _{\tau<\theta} \operatorname{dom}\left(\overrightarrow{S^{\tau}}\right)$. We define a $\bar{p}$-strategy $\vec{S}=\left\langle S_{i} \mid i \leq \alpha\right\rangle$ as follows. Fix $q \in W(\bar{p})$.

- For $i<\alpha, S_{i}(q)$ is defined as the unique element of $\left\{S_{i}^{\tau}(q) \mid \tau<\theta, i \in \operatorname{dom}\left(\overrightarrow{S^{\tau}}\right)\right\}$.

- For $i=\alpha$, we distinguish two cases:

$\longrightarrow$ If $S_{i}(q)=\varnothing$ for all $i<\alpha$, then we continue and let $S_{\alpha}(q):=\varnothing$;

$\gg$ Otherwise, let $S_{\alpha}(q):=\cup_{i<\alpha} S_{i}(q) \cup\left\{\beta_{q}\right\}$, where

$$
\beta_{q}:=\sup \left\{\max \left(S_{i}(q)\right) \mid i<\alpha, S_{i}(q) \neq \varnothing\right\} .
$$

Claim 6.15.1 $(\bar{p}, \vec{S}) \in A_{n}$. In particular, $(\bar{p}, \vec{S})$ is a lower bound for $D$. 
Proof Since, for each $\tau<\theta, \vec{S}^{\tau}$ is a $\bar{p}$-strategy, a moment of reflection makes it clear that we only need to verify that $S_{\alpha}$ is a labeled $\bar{p}$-tree. Let $q \in W(\bar{p})$ be arbitrary. As $\left\langle S_{i}(q)\right| i\langle\alpha\rangle$ is weakly ㄷ-increasing sequence of closed sets we only need to verify Clauses (3) and (4) of Definition 6.2. First we show that $q \Vdash_{\mathbb{P}} S_{\alpha}(q) \cap \dot{T}=\varnothing$. For this aim observe that Definition 6.2(4) yields $\left(q, \max \left(S_{i}(q)\right) \in R\right.$, for each $i<\alpha$. Now, for each $r \leq q$ with $\ell(r) \in I$ and $i<\alpha, r \Vdash_{\mathbb{P}_{\ell(r)}} \max \left(S_{i}(q)\right) \in \dot{C}_{\ell(r)}$, hence $r \Vdash_{\mathbb{P}_{\ell(r)}} \beta_{q} \in$ $\dot{C}_{\ell(r)}$, and thus, again by definition of $R,\left(\beta_{q}, q\right) \in R$ (cf. Lemma 5.6). Combining Definition 6.2(3) with $\left(\beta_{q}, q\right) \in R$, it altogether follows that $q \Vdash_{\mathbb{P}} S_{\alpha}(q) \cap \dot{T}=\varnothing$.

Finally let $q^{\prime} \leq q$ and let us check that the last bullet holds. For all $i<\alpha$, since $S_{i}$ is a $\bar{p}$-strategy, either $S_{i}\left(q^{\prime}\right)=\varnothing$ or $\left(\max \left(S_{i}\left(q^{\prime}\right)\right), q\right) \in R$. If $S_{\alpha}\left(q^{\prime}\right) \neq \varnothing$, then $\max \left(S_{\alpha}\left(q^{\prime}\right)\right)$ is the limit of $\left\langle\max \left(S_{i}\left(q^{\prime}\right)\right) \mid i<\alpha, S_{i}\left(q^{\prime}\right) \neq \varnothing\right\rangle$, so that, arguing as before, $\left(\max \left(S_{\alpha}\left(q^{\prime}\right)\right), q\right) \in R$.

Thus we have shown that $(\bar{p}, \vec{S}) \in A_{n}$ and clearly $(\bar{p}, \vec{S})$ gives a lower bound for $D$.

This completes the proof.

Lemma 6.16 (Mixing property) Let $(p, \vec{S})=a \in A, p^{\prime} \leq^{0} p$, and $m<\omega$. Suppose that $g: W_{m}\left(p^{\prime}\right) \rightarrow \mathbb{A} \downarrow$ a is a function such that $\pi \circ g$ is the identity map. Then there exists $b \unlhd^{0} a$ with $\pi(b)=p^{\prime}$ such that $h(b)(r) \unlhd^{0} g(r)$ for every $r \in W_{m}\left(p^{\prime}\right)$.

Proof Using Definition 2.3(5), we may find some cardinal $\theta<\mu$ and an injective enumeration $\left\{r^{\tau} \mid \tau<\theta\right\}$ of $W_{m}\left(p^{\prime}\right)$. For each $\tau<\theta$, let $\vec{S}^{\tau}$ be such that $g\left(r^{\tau}\right)=$ $\left(r^{\tau}, \vec{S}^{\tau}\right)$. As we are seeking $b \unlhd^{0} a$ such that, in particular, for every $\tau<\theta, \pitchfork(b)(r) \unlhd^{0}$ $g\left(r^{\tau}\right)$, we may make our life harder and assume that $\operatorname{dom}\left(\vec{S}^{\tau}\right)$ is nonzero, say $\operatorname{dom}\left(\vec{S}^{\tau}\right)=\alpha_{\tau}+1$.

Set $\alpha:=\sup (\operatorname{dom}(\vec{S}))$, so that, if $\operatorname{dom}(\vec{S})>0$, then $\operatorname{dom}(\vec{S})=\alpha+1$. Set $\alpha^{\prime}:=$ $\sup _{\tau<\theta} \alpha_{\tau}$, and note that, by regularity of $\mu, \alpha \leq \alpha^{\prime}<\mu$. Our goal is to define a sequence $\vec{T}=\left\langle T_{i}: W\left(p^{\prime}\right) \rightarrow[\mu]^{<\mu} \mid i \leq \alpha^{\prime}\right\rangle$ for which $b:=\left(p^{\prime}, \vec{T}\right)$ satisfies the conclusion of the lemma.

As $\left\{r^{\tau} \mid \tau<\theta\right\}$ is an enumeration of the $m^{\text {th }}$-level of the $p$-tree $W\left(p^{\prime}\right)$, Lemma 2.8 entails that, for each $q \in W\left(p^{\prime}\right)$, there is a unique ordinal $\tau_{q}<\theta$, such that $q$ is comparable with $r^{\tau_{q}}$. It thus follows from Lemma 2.8(3) that, for all $q \in W\left(p^{\prime}\right)$, $\ell(q)-\ell\left(p^{\prime}\right) \geq m$ iff $q \in W\left(r^{\tau_{q}}\right)$.

Now, for all $i \leq \alpha^{\prime}$ and $q \in W\left(p^{\prime}\right)$, let:

$$
T_{i}(q):= \begin{cases}S_{\min \left\{i, \alpha_{\tau_{q}}\right\}}^{\tau_{q}}(q), & \text { if } q \in W\left(r^{\tau_{q}}\right) ; \\ S_{\min \{i, \alpha\}}(w(p, q)), & \text { if } q \notin W\left(r^{\tau_{q}}\right) \text { and } \alpha>0 \\ \varnothing, & \text { otherwise. }\end{cases}
$$

Claim 6.16.1 Let $i \leq \alpha^{\prime}$. Then $T_{i}$ is a labeled $p^{\prime}$-tree.

Proof Fix $q \in W\left(p^{\prime}\right)$ and let us go over the Clauses of Definition 6.2.

(1) It is clear that in any of the three cases, $T_{i}(q)$ is a closed bounded subset of $\mu$.

(2) Let $q^{\prime} \leq q$. We focus on the non-trivial case in which $\ell\left(q^{\prime}\right)-\ell\left(p^{\prime}\right) \geq m$, while $\ell(q)-\ell\left(p^{\prime}\right)<m$ and $\alpha>0$. 
- If $i \leq \alpha$, then $T_{i}(q)=S_{i}(w(p, q))$ and $T_{i}\left(q^{\prime}\right)=S_{i}^{\tau_{q}}\left(q^{\prime}\right)$. In this case, since $w\left(r^{\tau_{q}}, q\right) \leq w(p, q)$ and $\vec{S}$ is a $p$-strategy, $S_{i}(w(p, q)) \subseteq S_{i}\left(w\left(r^{\tau_{q}}, q\right)\right)$. In addition, since $\left(r^{\tau_{q}}, \vec{S}^{\tau_{q}}\right) \unlhd(p, \vec{S}), S_{i}\left(w\left(r^{\tau_{q}}, q\right)\right)=S_{i}^{\tau_{q}}(q)$, so that $T_{i}(q) \subseteq S_{i}^{\tau_{q}}(q)$. But $S_{i}^{\tau_{q}}(q) \subseteq S_{i}^{\tau_{q}}\left(q^{\prime}\right)$, so that altogether $T_{i}(q) \subseteq T_{i}\left(q^{\prime}\right)$, as desired.

- If $i>\alpha$, then $T_{i}(q)=S_{\alpha}(w(p, q))$ and $T_{i}\left(q^{\prime}\right)=S_{j}^{\tau_{q}}\left(q^{\prime}\right)$ for $j:=\min \left\{i, \alpha_{\tau_{q}}\right\}$. In this case, as $\vec{S}$ is a $p^{\prime}$-strategy and $\vec{S}^{\tau_{q}}$ is an $r^{\tau_{q}}$-strategy, we infer from $\left(r^{\tau_{q}}, \vec{S}^{\tau_{q}}\right) \unlhd(p, \vec{S})$ that:

$$
S_{\alpha}(w(p, q)) \subseteq S_{\alpha}\left(w\left(r^{\tau_{q}}, q\right)\right)=S_{\alpha}^{\tau_{q}}(q) \subseteq S_{j}^{\tau_{q}}(q) \subseteq S_{j}^{\tau_{q}}\left(q^{\prime}\right) .
$$

Altogether, $T_{i}(q) \subseteq T_{i}\left(q^{\prime}\right)$, as desired.

(3) If $q \in W\left(r^{\tau_{q}}\right)$, then this follows from the fact that $S_{\min \left\{i, \alpha_{\tau_{q}}\right\}}^{\tau_{q}}$ is a labeled $r^{\tau_{q}}$-tree. If $q \notin W\left(r^{\tau_{q}}\right)$ and $\alpha>0$, then this follows from the fact that $S_{\min \{i, \alpha\}}$ is a labeled $p$-tree and $q \leq w(p, q)$.

(4) Let $q^{\prime} \leq q$ in $W\left(p^{\prime}\right)$ and assume that $T_{i}\left(q^{\prime}\right) \neq \varnothing$. We focus on the case $T_{i}\left(q^{\prime}\right)=$ $S_{j}\left(w\left(p, q^{\prime}\right)\right)$, for $j:=\min \{i, \alpha\}$. In particular, $\beta:=\max \left(S_{j}\left(w\left(p, q^{\prime}\right)\right)\right)$ is welldefined. Clearly $w\left(p, q^{\prime}\right) \leq w(p, q)$ so, since $S_{j}$ is a labeled $p$-tree, $\left(\beta, w\left(p, q^{\prime}\right)\right) \epsilon$ $R$. But $q^{\prime} \leq w\left(p, q^{\prime}\right)$, so by the nature of $R$, we have that $\left(\beta, q^{\prime}\right) \in R$, as well.

Claim 6.16.2 The sequence $\vec{T}=\left\langle T_{i}: W\left(p^{\prime}\right) \rightarrow[\mu]^{<\mu} \mid i \leq \alpha^{\prime}\right\rangle$ is a $p^{\prime}$-strategy.

Proof We need to go over the clauses of Definition 6.3. However, Clause (1) is trivial, Clause (2) is established in the preceding claim, and Clauses (3) and (5) follow from the corresponding features of $\vec{S}$ and the $\vec{S}^{\tau}$ 's. Thus, we are left with verifying Clause (4).

To this end, fix $i<\alpha$ and a pair $q^{\prime} \leq q$ in $W\left(p^{\prime}\right)$. We have to show that $\left(T_{i+1}(q) \backslash T_{i}(q)\right) \sqsubseteq\left(T_{i+1}\left(q^{\prime}\right) \backslash T_{i}\left(q^{\prime}\right)\right)$. As before, the only non-trivial case is when $\ell\left(q^{\prime}\right)-\ell\left(p^{\prime}\right) \geq m$, while $\ell(q)-\ell\left(p^{\prime}\right)<m$ and $\alpha>0$. To avoid arguing about the empty set, we may also assume that $\alpha>i$. In particular, $\alpha_{\tau}>i$. So

- $T_{i+1}(q) \backslash T_{i}(q)=S_{i+1}(w(p, q)) \backslash S_{i}(w(p, q))$, and

- $T_{i+1}\left(q^{\prime}\right) \backslash T_{i}\left(q^{\prime}\right)=S_{i+1}^{\tau_{q}}\left(q^{\prime}\right) \backslash S_{i}^{\tau_{q}}\left(q^{\prime}\right)$.

Now, as $\vec{S}$ is a $p$-strategy, we infer that $S_{i+1}(w(p, q)) \backslash S_{i}(w(p, q)) \sqsubseteq S_{i+1}$ $\left(w\left(p, q^{\prime}\right)\right) \backslash S_{i}\left(w\left(p, q^{\prime}\right)\right)$. But $\left(r^{\tau} q^{\prime}, \vec{S}^{\tau} q^{\prime}\right) \unlhd(p, \vec{S})$, and hence, for each $j \in\{i, i+1\}$, $S_{j}^{\tau} \tau_{q^{\prime}}\left(q^{\prime}\right)=S_{j}\left(w\left(p, q^{\prime}\right)\right)$. The desired equation now follows immediately.

Thus, we have established that $b:=\left(p^{\prime}, \vec{T}\right)$ is a legitimate condition.

Claim 6.16.3 $\pi(b)=p^{\prime}$ and $b \unlhd^{0} a$.

Proof The first assertion is trivial, and it also implies that $b \unlhd^{0} a$ iff $b \unlhd a$, hence, we focus on establishing the latter. As $p^{\prime} \leq p$ and $\alpha^{\prime} \geq \alpha$, we are left with verifying Clause (2c) of Definition 6.4. To avoid trivialities, suppose also that $\alpha>0$. Now, let $i \leq \alpha$ and $q \in W\left(p^{\prime}\right)$ be arbitrary.

- If $\ell(q)<\ell\left(p^{\prime}\right)+m$, then we have $T_{i}(q)=S_{i}(w(p, q))$, and we are done.

- If $\ell(q) \geq \ell\left(p^{\prime}\right)+m$, then $T_{i}(q)=S_{i}^{\tau_{q}}(q)$ and, since $\left(r^{\tau_{q}}, \vec{S}^{\tau_{q}}\right) \unlhd(p, \vec{S}), T_{i}(q)=$ $S_{i}(w(p, q))$, as desired.

Claim 6.16.4 Let $\tau<\theta$. For each $q \in W\left(r^{\tau}\right), w\left(p^{\prime}, q\right)=w\left(r^{\tau}, q\right)$. 
Proof As $r^{\tau} \leq p^{\prime}$, we have $\left\{s \mid q \leq s \leq r^{\tau}\right\} \subseteq\left\{s \mid q \leq s \leq p^{\prime}\right\}$, so that $w\left(r^{\tau}, q\right) \leq$ $w\left(p^{\prime}, q\right)$. In addition, as $w\left(p^{\prime}, q\right)$ and $r^{\tau}$ are compatible elements of $W\left(p^{\prime}\right)$ (as witnessed by $q)$, we infer from Lemma 2.8(2), $\ell\left(w\left(p^{\prime}, q\right)\right)=\ell(q) \geq \ell\left(r^{\tau}\right)$ and Definition 2.3(1), that $w\left(p^{\prime}, q\right) \leq r^{\tau}$, so that $w\left(p^{\prime}, q\right) \in\left\{s \mid q \leq s \leq r^{\tau}\right\}$, and hence $w\left(p^{\prime}, q\right) \leq$ $w\left(r^{\tau}, q\right)$.

Recalling Claim 6.16.3, to complete our proof, we fix an arbitrary $\tau<\theta$, and turn to show that $h(b)\left(r^{\tau}\right) \unlhd^{0} g\left(r^{\tau}\right)$. By Lemma 6.13(5), $\pi\left(\pitchfork(b)\left(r^{\tau}\right)\right)=r^{\tau}=\pi\left(g\left(r^{\tau}\right)\right)$, so that we may focus on verifying that $\pitchfork(b)\left(r^{\tau}\right) \unlhd g\left(r^{\tau}\right)$.

To this end, let $\vec{T}^{\tau}$ denote the $r^{\tau}$-strategy such that $\downarrow(b)\left(r^{\tau}\right)=\left(r^{\tau}, \vec{T}^{\tau}\right)$. By Definition 6.11(*), $\operatorname{dom}\left(\vec{T}^{\tau}\right)=\operatorname{dom}(\vec{T})=\alpha^{\prime}+1$, hence $\operatorname{dom}\left(\vec{S}^{\tau}\right)=\alpha_{\tau}+1 \leq \alpha^{\prime}+1 \leq$ $\operatorname{dom}\left(\vec{T}^{\tau}\right)$. Now, let $i \leq \alpha_{\tau}$ and $q \in W\left(r^{\tau}\right)$. By Definition 6.11(*), $T_{i}^{\tau}(q)=T_{i}\left(w\left(p^{\prime}, q\right)\right)$. By the preceding claim $w\left(p^{\prime}, q\right)=w\left(r^{\tau}, q\right)$, so that $q^{\prime}:=w\left(p^{\prime}, q\right)$ is in $W\left(r^{\tau}\right)$ and $\tau_{q^{\prime}}=\tau$. In effect, by definition of $T_{i}\left(q^{\prime}\right)$ (just before Claim 6.16.1, we get that $T_{i}\left(q^{\prime}\right)=$ $S_{i}^{\tau}\left(q^{\prime}\right)$. Altogether, $T_{i}^{\tau}(q)=S_{i}^{\tau}\left(q^{\prime}\right)=S_{i}^{\tau}\left(w\left(r^{\tau}, q^{\prime}\right)\right)$, as required by Clause (2c) of Definition 6.4.

Corollary $6.17\left(\mathbb{A}, \ell_{\mathbb{A}}, c_{\mathbb{A}}\right)$ is a $\sum$-Prikry triple, and $1_{\mathbb{A}} \Vdash_{\mathbb{A}} \check{\mu}=\check{\kappa}^{+}$.

Proof We first go over the clauses of Definition 2.3:

(1) By Lemma 4.5.

(2) By Lemma 4.6 together with Lemma 6.15.

(3) By Lemma 4.7 and the fact that $\left|H_{\mu}\right|=\mu$.

(4) By Lemma 4.8.

(5) By Lemma 4.9.

(6) By Lemma 4.10.

(7) By Lemma 4.12 together with Lemma 6.16.

Finally, by Corollary 4.13 and the fact that $1_{\mathbb{P}} \Vdash_{\mathbb{P}}$ “ $\check{\kappa}$ is singular, " $1_{\mathbb{A}} \Vdash_{\mathbb{A}} \check{\mu}=\check{\kappa}^{+}$.

For the record, we make explicit one more feature of the forking projection constructed in this section.

Lemma 6.18 (Transitivity) Let $a \in A$. For all $q \leq \pi(a)$ and $r \in W(q)$,

$$
\pitchfork(a)(r)=\hbar(h(a)(q))(r) .
$$

Proof Set $(p, \vec{S}):=a$. Fix an arbitrary $q \leq \pi(a)$, and let $b=\hbar(a)(q)$. Fix an arbitrary $r \in W(q)$, and set $(t, \vec{T}):=\pitchfork(a)(r)$ and $(u, \vec{U}):=\pitchfork(b)(r)$. By Definition 6.11, it follows that $t=r=u$ and $\operatorname{dom}(\vec{T})=\operatorname{dom}(\vec{S})=\operatorname{dom}(\vec{U})$. Once again Definition 6.11 yields, for each $i \in \operatorname{dom}(\vec{S})$ and $s \in W(t), T_{i}(s)=S_{i}(w(p, s))$. Analogously, for each $i \in \operatorname{dom}(\vec{S})$ and $s \in W(u), Q_{i}(s)=S_{i}(w(p, s))$. Altogether, $W(t)=W(u)$, and for each $i \in \operatorname{dom}(\vec{S})$ and $s \in W(u), T_{i}(s)=Q_{i}(s)$, as desired.

\section{Conclusion}

By putting everything together, we arrive at the following corollary:

Corollary 7.1 Suppose $\Sigma=\left\langle\kappa_{n} \mid n<\omega\right\rangle$ is a non-decreasing sequence of Laverindestructible supercompact cardinals, and let $\kappa:=\sup (\Sigma)$. Suppose: 
(i) $(\mathbb{P}, \ell, c)$ is a $\sum$-Prikry notion of forcing, and $\mathbb{1}_{\mathbb{P}} \mathbb{P}_{\mathbb{P}}$ " $\kappa$ is singular";

(ii) $1_{\mathbb{P}} \Vdash \mathbb{P} \check{\mu}=\check{\kappa}^{+}$, for some cardinal $\mu=\mu^{<\mu}$;

(iii) $\mathbb{P} \subseteq H_{\mu^{+}}$;

(iv) $r^{\star} \in P$ forces that $z$ is a $\mathbb{P}$-name for a stationary subset of $\left(E_{\omega}^{\mu}\right)^{V}$ that does not reflect in $\left\{\alpha<\mu \mid \omega<\mathrm{cf}^{V}(\alpha)<\kappa\right\}$.

Then, there exists a $\Sigma$-Prikry triple $\left(\mathbb{A}, \ell_{\mathbb{A}}, c_{\mathbb{A}}\right)$ such that:

(1) $\left(\mathbb{A}, \ell_{\mathbb{A}}, c_{\mathbb{A}}\right)$ admits a forking projection to $(\mathbb{P}, \ell, c)$ that has the mixing property;

(2) $1_{\mathbb{A}} \Vdash \vdash_{\mathbb{A}} \check{\mu}=\check{\kappa}^{+}$;

(3) $\mathbb{A} \subseteq H_{\mu^{+}}$;

(4) $\left\lceil r^{\star}\right\rceil^{\mathbb{A}}$ forces that $z$ is nonstationary.

Proof By Lemma 5.8, for all $n<\omega, V^{\mathbb{P}_{n}} \vDash \operatorname{Refl}\left(<\omega, E_{<\kappa_{n}}^{\mu}, E_{<\kappa_{n}}^{\mu}\right)$. So, all the blanket assumptions of Section 6 are satisfied, and we obtain a notion of forcing $\mathbb{A}:=\mathbb{A}(\mathbb{P}, z)$ together with maps $\ell_{\mathbb{A}}$ and $c_{\mathbb{A}}$ such that, by Corollary 6.17, $\left(\mathbb{A}, \ell_{\mathbb{A}}, c_{\mathbb{A}}\right)$ is $\sum$-Prikry.

Now, Clauses (1) and (2) follow from Lemma 6.13 and Corollary 6.17, Clause (3) follows from Lemma 6.6, and Clause (4) follows from Theorem 6.8.

\section{References}

[1] O. Ben-Neria, Y. Hayut, and S. Unger, Statinoary reflection and the failure of SCH. Preprint, 2019. arXiv: 1908.11145

[2] P. J. Cohen, The independence of the continuum hypothesis. Proc. Natl. Acad. Sci. 50(1963), 1143-1148. https://doi.org/10.1073/pnas.50.6.1143

[3] P. J. Cohen, The independence of the continuum hypothesis. II. Proc. Natl. Acad. Sci. 51(1964), 105-110. https://doi.org/10.1073/pnas.51.1.105

[4] J. Cummings, Iterated forcing and elementary embeddings. In: Handbook of set theory, Vols. 1-3, Springer, Dordrecht, The Netherlands, 2010, pp. 775-883. http://dx.doi.org/10.1007/978-1-4020-5764-9_13

[5] J. Cummings, M. Džamonja, M. Magidor, C. Morgan, and S. Shelah, A framework for forcing constructions at successors of singular cardinals. Trans. Am. Math. Soc. 369(2017), no. 10, 7405-7441. https://doi.org/10.1090/tran/6974

[6] J. Cummings, M. Foreman, and M. Magidor, Squares, scales and stationary reflection. J. Math. Log. 1(2001), no. 1, 35-98. https://doi.org/10.1142/s021906130100003x

[7] J. Cummings, S.-D. Friedman, M. Magidor, A. Rinot, and D. Sinapova, Ordinal definable subsets of singular cardinals. Israel J. Math. 226(2018), no. 2, 781-804. https://doi.org/10.1007/s11856-018-1712-2

[8] M. Dzamonja and S. Shelah, Universal graphs at the successor of a singular cardinal. J. Symb. Log. 68(2003), 366-388. https://doi.org/10.2178/jsl/1052669056

[9] T. Eisworth, On iterated forcing for successors of regular cardinals. Fundam. Math. 179(2003), no. 3, 249-266. https://doi.org/10.4064/fm179-3-4

[10] D. H. Fremlin, Consequences of Martin's axiom. Cambridge Tracts in Mathematics, 84, Cambridge University Press, Cambridge, MA, 1984. https://doi.org/10.1017/CBO9780511896972

[11] M. Gitik, Prikry-type forcings. In: Handbook of set theory, Vols. 1-3, Springer, Dordrecht, The Netherlands, 2010, pp. 1351-1447. https://doi.org/10.1007/978-1-4020-5764-9_17

[12] M. Gitik and M. Magidor, Extender based forcings. J. Symb. Log. 59(1994), no. 2, 445-460. https://doi.org/10.2307/2275399

[13] M. Gitik and A. Rinot, The failure of diamond on a reflecting stationary set. Trans. Am. Math. Soc. 364(2012), no. 4, 1771-1795. https://doi.org/10.1090/s0002-9947-2011-05355-9

[14] M. Gitik and A. Sharon, On SCH and the approachability property. Proc. Am. Math. Soc. 136(2008), no. 1, 311-320. https://doi.org/10.1090/s0002-9939-07-08716-3

[15] K. Gödel, The consistency of the continuum hypothesis. Annals of Mathematics Studies, 3, Princeton University Press, Princeton, NJ, 1940. 
[16] A. Kanamori, The higher infinite. Large cardinals in set theory from their beginnings. 2nd ed. Springer Monographs in Mathematics, Springer-Verlag, Berlin, Germany, 2009. Paperback reprint of the 2003 edition.

[17] C. Lambie-Hanson and A. Rinot, Knaster and friends I: Closed colorings and precalibers. Algebr. Univ. 79(2018), no. 4, 39, Art. 90. https://doi.org/10.1007/s00012-018-0565-1

[18] A. Poveda, A. Rinot, and D. Sinapova, Sigma-Prikry forcing II: iteration scheme. J. Math. Log., to appear. http://assafrinot.com/paper/42

[19] A. Rinot, Chain conditions of products, and weakly compact cardinals. Bull. Symb. Log. 20(2014), no. 3, 293-314. https://doi.org/10.1017/bsl.2014.24

[20] A. Rosłanowski, Explicit example of collapsing $\kappa^{+}$in iteration of $\kappa$-proper forcings. Preprint, 2018. arXiv:1808.01636

[21] A. Rosłanowski and S. Shelah, The last forcing standing with diamonds. Fundam. Math. 246(2019), no. 2, 109-159. https://doi.org/10.4064/fm898-9-2018

[22] A. Rosłanowski and S. Shelah, Iteration of $\lambda$-complete forcing notions not collapsing $\lambda^{+}$. Int. J. Math. Math. Sci. 28(2001), 63-82. https://doi.org/10.1155/s016117120102018x

[23] A. Rosłanowski and S. Shelah, Lords of the iteration. In: Set theory and its applications, Contemporary Mathematics (CONM), 533, Amer. Math. Soc., Providence, RI, 2011, pp. 287-330. https://doi.org/10.1090/conm/533/10514

[24] A. Rosłanowski and S. Shelah, More about $\lambda$-support iterations of $(<\lambda)$-complete forcing notions. Arch. Math. Log. 52(2013), 603-629. https://doi.org/10.1007/s00153-013-0334-y

[25] A. Sharon, Weak squares, scales, stationary reflection and the failure of SCH. Ph.D. thesis, Tel Aviv University, Tel Aviv, 2005.

[26] S. Shelah, A weak generalization of MA to higher cardinals. Israel J. Math. 30(1978), 297-306. https://doi.org/10.1007/bf02761994

[27] S. Shelah, Diamonds, uniformization. J. Symb. Log. 49(1984), 1022-1033. https://doi.org/10.2307/2274258

[28] S. Shelah, Cardinal arithmetic. Oxford Logic Guides, 29, The Clarendon Press Oxford University Press, Oxford Science Publications, New York, NY, 1994.

[29] S. Shelah, Not collapsing cardinals $\leq \kappa$ in $(<\kappa)$-support iterations. Israel J Math. 136(2003), 29-115. https://doi.org/10.1007/bf02807192

[30] S. Shelah, Successor of singulars: combinatorics and not collapsing cardinals $\leq \kappa$ in $(<\kappa)$-support iterations. Israel J. Math. 134(2003), 127-155. https://doi.org/10.1007/bf02787405

[31] R. M. Solovay and S. Tennenbaum, Iterated Cohen extensions and Souslin's problem. Ann. Math. 94(1971), no. 2, 201-245. https://doi.org/10.2307/1970860

Departament de Matemàtiques i Informàtica, Universitat de Barcelona. Gran Via de les Corts Catalanes, Barcelona 585, 08007, Catalonia

e-mail: alejandro.poveda@ub.edu

Department of Mathematics, Bar-Ilan University, Ramat-Gan 5290002, Israel

e-mail: rinotas@math.biu.ac.il

URL: http://www.assafrinot.com

Department of Mathematics, Statistics, and Computer Science University of Illinois at Chicago, Chicago, IL 60607-7045, USA

e-mail:sinapova@uic.edu

URL: https://homepages.math.uic.edu/ sinapova/ 